\section{FUS-ALS mutants alter FMRP phase separation equilibrium and impair protein translation}

\author{
Nicol Birsa ${ }^{1,2 * \dagger}$, Agnieszka M. Ule ${ }^{1 \dagger}$, Maria Giovanna Garone ${ }^{3,4 \ddagger}$, Brian Tsang ${ }^{5,6 \ddagger}$,

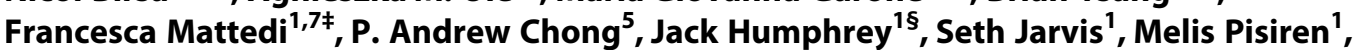 \\ Oscar G. Wilkins ${ }^{1,8}$, Micheal L. Nosella ${ }^{5}$, Anny Devoy ${ }^{9}$, Cristian Bodo ${ }^{1}$, Rafaela Fernandez de la Fuente ${ }^{1}$, \\ Elizabeth M. C. Fisher' ${ }^{1}$, Alessandro Rosa ${ }^{3,4}$, Gabriella Viero ${ }^{7}$, Julie D. Forman-Kay ${ }^{5,6}$, \\ Giampietro Schiavo ${ }^{1,2}$, Pietro Fratta ${ }^{1,10_{*}}$
}

FUsed in Sarcoma (FUS) is a multifunctional RNA binding protein (RBP). FUS mutations lead to its cytoplasmic mislocalization and cause the neurodegenerative disease amyotrophic lateral sclerosis (ALS). Here, we use mouse and human models with endogenous ALS-associated mutations to study the early consequences of increased cytoplasmic FUS. We show that in axons, mutant FUS condensates sequester and promote the phase separation of fragile X mental retardation protein (FMRP), another RBP associated with neurodegeneration. This leads to repression of translation in mouse and human FUS-ALS motor neurons and is corroborated in vitro, where FUS and FMRP copartition and repress translation. Last, we show that translation of FMRP-bound RNAs is reduced in vivo in FUS-ALS motor neurons. Our results unravel new pathomechanisms of FUS-ALS and identify a novel paradigm by which mutations in one RBP favor the formation of condensates sequestering other RBPs, affecting crucial biological functions, such as protein translation.

\section{INTRODUCTION}

The fate of mRNAs in the cytoplasm, including their localization, stability and translation, is regulated by RNA binding proteins (RBPs). These RBPs often contain low-complexity regions (LCRs) that promote their phase separation into biomolecular condensates. RNA transport granules and stress granules are two examples of biomolecular condensates and their dynamics, such as trafficking, assembly and disassembly, impact on the availability, localization, and translation of bound RNAs.

FUsed in Sarcoma (FUS) is an LCR-containing RBP involved in various aspects of RNA metabolism. Under physiological conditions, FUS is predominantly localized in the nucleus where it is involved in transcription, mRNA processing, and miRNA biogenesis (1); however, low levels of the protein are also present in the cytoplasm (2). FUS mutations are causative of amyotrophic lateral sclerosis (ALS), a relentless neurodegenerative disorder in which the loss of motor neurons (MNs) leads to a progressive impairment of the neuromuscular system. Most FUS mutations disrupt the C-terminal nuclear localization signal (NLS), leading to a nuclear depletion and a cytoplasmic mislocalization of FUS (3). The loss of

\footnotetext{
'Department of Neuromuscular Diseases, UCL Queen Square Institute of Neurology, London WC1N 3BG, UK. ${ }^{2}$ UK Dementia Research Institute, University College London, London WC1E 6BT, UK. ${ }^{3}$ Department of Biology and Biotechnology Charles Darwin, Sapienza University of Rome, P.le A. Moro 5, 00185 Rome, Italy. ${ }^{4}$ Center for Life Nano Science, Istituto Italiano di Tecnologia, Viale Regina Elena 291, 00161 Rome, Italy. ${ }^{5}$ Program in Molecular Medicine, The Hospital for Sick Children, Toronto, ON M5G 0A4, Canada. ${ }^{6}$ Department of Biochemistry, University of Toronto, Toronto, ON M5S 1A8, Canada. Institute of Biophysics, CNR, Trento, Italy. ${ }^{8}$ The Francis Crick Institute, London NW1 1AT, UK. ${ }^{9}$ Maurice Wohl Clinical Neuroscience Institute, King's College London, London SE5 9RT, UK. ${ }^{10}$ MRC Centre for Neuromuscular Disease, Queen Square, London WC1N 3BG, UK.

*Corresponding author. Email: p.fratta@ucl.ac.uk (P.F.); n.birsa@ucl.ac.uk (N.B.)

tThese authors contributed equally to this work.

₹These authors contributed equally to this work.

§Present address: Icahn School of Medicine at Mount Sinai, 1 Gustave L. Levy PI, New York, NY 10029, USA
}

FUS nuclear RNA processing functions, however, is not sufficient to induce neurodegeneration on its own, drawing attention to the cytoplasmic roles of FUS and how these are affected by its increased cytoplasmic levels, which ultimately result in cytoplasmic neuronal aggregates in patient post mortem tissue $(4,5)$.

The role of FUS in the cytoplasm and the functional consequences of ALS-linked mutations are poorly understood; however, an increasing number of studies suggest that FUS liquid-liquid phase separation (LLPS) properties play a crucial role in its cytoplasmic gain of function (6-8). Under physiological conditions, the propensity of FUS to undergo LLPS in the cytoplasm is limited by its low concentration; in contrast, the increased cytoplasmic localization of FUS mutants favors the transition to a phase-separated state. Moreover, decreased interaction with the nuclear import factor and chaperone TNPO1, posttranslational modifications and intrinsic properties given by ALS mutations all contribute to the formation of cytoplasmic FUS condensates $(6,7,9,10)$, the biology and composition of which are, to date, poorly understood. Potential disease mechanisms have been identified in various overexpression systems $(7,11)$, although it remains unclear how well these model the physiological setting, where the expression of FUS, as well as most RBPs, is finely tuned both by auto- and cross-regulatory mechanisms (12).

Here, we use mouse and human models with endogenous expression of FUS-ALS mutations and show that mutant FUS forms cytoplasmic condensates containing the RBP fragile X mental retardation protein (FMRP). FUS and FMRP repress de novo protein synthesis in vitro and in MNs. We find that this is not caused by direct interaction with the translational machinery; instead, our data support a model whereby FUS and FMRP condensates have an antagonistic role to translation, resulting in decreased ribosome occupancy of FMRP mRNA targets. Our findings highlight how mutant FUS can posttranscriptionally alter RBP dynamics and function. 


\section{RESULTS}

\section{FUS-ALS mutants increase axonal FMRP condensates}

To investigate the consequences of ALS-causative mutations on FUS cytoplasmic function, we used the $\Delta 14$ FUS knockin mouse model (13), in which a mutation causing aggressive and early-onset ALS (14) leads to skipping of exon 14 and a frameshift in exon 15. This frameshift leads to the loss of most of the RGG3 domain, the complete deletion of the NLS, and the formation of a unique C-terminal peptide sequence (fig. S1A), allowing the generation of mutant-specific antibodies (13). Similarly to $\mathrm{Fus}^{-/-}$mice (4), Fus ${ }^{\Delta 14 / \Delta 14}$ mice die perinatally on a congenic background, likely because of respiratory failure. To investigate the function of $\Delta 14$ FUS in MNs, as well as its dosage-dependent effects, we used both heterozygous and homozygous primary embryonic MNs. While wild-type FUS has a predominantly nuclear localization in both $\mathrm{Fus}^{+/+}$and $\mathrm{Fus}{ }^{\Delta 14 /+} \mathrm{MNs}, \Delta 14$ FUS is enriched in the cytoplasm of Fus ${ }^{\Delta 14 /+}$ and Fus ${ }^{\Delta 14 / \Delta 14}$ neurons, is distributed across cell body and neurites (Fig. 1, A and B, and fig. $\mathrm{S} 1, \mathrm{~B}$ to $\mathrm{D})$, and can be detected in a punctate, condensate-like form (Fig. 1, A and B, and fig. S1B). Notably, $\Delta 14$ FUS expression in Fus ${ }^{\Delta 14 /+}$ MNs does not induce the mislocalization of wild-type FUS (Fig. 1A and fig. S1, B and C).

FUS interacts with several other RBPs, including FMRP and Survival Motor Neuron (SMN), two well-characterized RBPs that, similarly to FUS, are present in cytoplasmic RNA granules and are strongly linked to neurological diseases (15-20). In addition, FMRP has been detected in FUS-positive inclusions in post mortem tissue (15). This prompted us to test whether physiological expression of mutant FUS could affect these RBPs. FMRP and SMN interact with both wild-type and $\Delta 14$ FUS (fig. S2A). To investigate their localization, we focused on MN axons where both RBPs are incorporated into RNA transport granules. The density of axonal FMRP puncta was increased in primary Fus ${ }^{\Delta 14 /+}$ and Fus ${ }^{\Delta 14 / \Delta 14}$ MNs compared to controls in a mutant FUS dosage-dependent manner (Fig. 1, C and D, and fig. S2B; normalized axonal FMRP puncta density in $\mathrm{Fus}^{+/+}=100 \pm 5.2, \mathrm{Fus}^{\Delta 14 /+}=$ $154.2 \pm 8.2$, and Fus $\left.{ }^{\Delta 14 / \Delta 14}=181.5 \pm 9.2,{ }^{* * *} P<0.0001\right)$. In contrast, no significant changes in the somatic intensity of FMRP staining or in the total FMRP expression in MN cultures were detected (Fig. 1G and fig. S2, D to F; somatic FMRP fluorescence intensity in $\mathrm{Fus}^{+/+}=1 \pm 0.04$, Fus $^{\Delta 14 /+}=1.2 \pm 0.05$, and Fus ${ }^{\Delta 14 / \Delta 14}=1.0 \pm 0.07$; FMRP levels in cultured MN lysates Fus ${ }^{+/+}=1.9 \pm 0.45, \mathrm{Fus}^{\Delta 14 /+}=$ $1.9 \pm 0.4$, and $F u s^{\Delta 14 / \Delta 14}=1.7 \pm 0.4$ ). Puncta size was also unaffected (fig. S2C; average FMRP axonal puncta size in $\mathrm{Fus}^{+/+}=0.26 \pm 0.01 \mu \mathrm{m}^{2}$, Fus $^{\Delta 14 /+}=0.28 \pm 0.01 \mu \mathrm{m}^{2}$, and Fus $s^{\Delta 14 / \Delta 14}=0.29 \pm 0.01 \mu \mathrm{m}^{2}$ ).

FUS P525L is a well-described mutation that impairs the PY-NLS, leading to the cytoplasmic mislocalization of FUS (fig. S2G). Similar to the mouse primary MNs, we also observed an $80 \%$ increase in FMRP axonal puncta in human induced pluripotent stem cell (iPSC)-derived FUS ${ }^{P 525 L / P 525 L}$ MNs (normalized axonal FMRP puncta density in isogenic control $=100 \pm 4.4$, FUS $^{P 525 L / P 525 L}=$ $182.5 \pm 5.8,{ }^{* * *} P<0.0001$; Fig. $1, \mathrm{H}$ and I). FMRP puncta colocalized with FUS in both primary and iPSC-derived MNs (Fig. 1, E and F, and fig. S2H), with $\sim 60 \%$ of FMRP puncta either fully or partially positive for mutant FUS (percentage of $\Delta 14$ FUS-positive FMRP puncta: $\mathrm{Fus}^{\mathrm{\Delta 14/+}}$ axons, $16.3 \%$ full overlap and $39.1 \%$ partial overlap; Fus ${ }^{\Delta 14 / \Delta 14}$ axons, $20.6 \%$ full overlap and $45.4 \%$ partial overlap; Fig. 1, E and F), suggesting a primary role for FUS in altering FMRP dynamics. We found minimal alterations in FMRP puncta density in FUS knockout axons (fig. S2, I and J; normalized axonal FMRP puncta density in $\mathrm{Fus}^{+/+}=100 \pm 4.1, \mathrm{Fus}^{+/-}=77.26 \pm 5.5$, and
$\mathrm{Fus}^{-/-}$axons $\left.=85.9 \pm 5.7\right)$, supporting that the increase in axonal FMRP puncta is caused by a gain of function of mutant FUS.

ALS-associated FUS mutants alter SMN dynamics, particularly its localization to nuclear gems $(4,16-18)$. We therefore investigated whether mutant FUS expression also affects SMN distribution along axons. We found that SMN puncta density was unaltered in Fus ${ }^{\Delta 14 /+}$ and $\mathrm{Fus}{ }^{\Delta 14 / \Delta 14} \mathrm{MN}$ axons compared to $\mathrm{Fus}^{+/+}$axons (fig. S2, K and $\mathrm{L}$; normalized axonal SMN puncta density in $\mathrm{Fus}^{+/+}=100 \pm 5.2$, Fus $^{\Delta 14 /+}=101.1 \pm 5.9$, and Fus ${ }^{\Delta 14 / \Delta 14}=122.0 \pm 7.9$ ), suggesting that only a subset of FUS-binding RBPs, such as FMRP, are affected by mutant FUS.

To assess whether a general impairment in axonal transport could account for the altered FMRP distribution, we analyzed the density of lysosome-associated membrane protein 1 (LAMP1)-positive organelles in MN axons. Mutant FUS expression did not significantly affect the density of these structures within this compartment (fig. S3, $\mathrm{A}$ and B; normalized axonal LAMP1 puncta density in $\mathrm{Fus}^{+/+}=100 \pm$ 6.5, Fus $^{\Delta 14 /+}=112.6 \pm 6.22$, and Fus ${ }^{\Delta 14 / \Delta 14}=123.2 \pm 8.1$ ). Moreover, we analyzed the axonal transport of both signaling endosomes and mitochondria, and we found it to be unaffected in $\triangle 14$ FUS MNs at 7 days in vitro (fig. S3, C to E; signaling endosome average track velocity in Fus ${ }^{+/+}=1.4 \pm 0.08 \mu \mathrm{m} / \mathrm{s}$, Fus $^{\Delta 14 /+}=1.5 \pm 0.1 \mu \mathrm{m} / \mathrm{s}$, and $\mathrm{Fus}^{\Delta 14 / \Delta 14}=1.4 \pm 0.09 \mu \mathrm{m} / \mathrm{s}$; time spent pausing in $\mathrm{Fus}^{+/+}=$ $17.1 \pm 1.9 \%$, Fus $^{\Delta 14 /+}=11.9 \pm 0.9 \%$, and Fus ${ }^{\Delta 14 / \Delta 14}=13.61 \pm 1.4 \%$; mitochondria average velocity in Fus ${ }^{+/+}=0.7 \pm 0.06 \mu \mathrm{m} / \mathrm{s}, \mathrm{Fus}^{\Delta 14 /+}=$ $0.6 \pm 0.05 \mu \mathrm{m} / \mathrm{s}$, and Fus ${ }^{\Delta 14 / \Delta 14}=0.6 \pm 0.06 \mu \mathrm{m} / \mathrm{s}$ ), in agreement with recently published data (21).

\section{FMRP partitions into FUS condensates}

To further explore how mutant FUS induces FMRP granules, we asked whether the two proteins could cophase separate in vitro. We performed an in vitro LLPS assay in which the disordered N-terminal region of FUS conjugated with fluorescein isothiocyanate (FITC) ( ${ }^{\text {FITC }}{ }_{\text {FUS }}{ }_{\mathrm{LCR}}$ ) was coincubated with the disordered C-terminal region of FMRP conjugated with Alexa Fluor 647 ( $\left.{ }^{\text {Alexa647 }}{ }^{F M R P} P_{L C R}\right)$, in the presence of sc1, a G-quadruplex-forming RNA known to bind FMRP $(22,23)$. The observed droplets were positive for both ${ }^{\text {IITC }_{F U S}}$ LCR $_{\mathrm{LC}}$ and ${ }^{A l e x a 647}{ }^{F M R P} P_{\text {LCR }}$ (Fig. 2A), evidence that the FUS disordered $\mathrm{N}$-terminal region phase separates with scl RNA and FMRP.

To analyze the partitioning of FMRP into droplets of full-length FUS, we generated wild-type, $\mathrm{P} 525 \mathrm{~L}$, and $\Delta 14$ recombinant FUS, fused to maltose binding protein (MBP) to enhance solubility and circumvent the strong propensity of recombinant FUS to aggregate (fig. S4A). Upon cleavage of the MBP tag by Tobacco Etch Virus (TEV) protease, all FUS proteins phase-separated in vitro as detected by turbidity measurements (Fig. 2B). FUS ${ }^{\Delta 14}$ displayed a lower LLPS propensity compared to FUS ${ }^{\mathrm{WT}}$ and FUS ${ }^{\mathrm{P} 525 \mathrm{~L}}$, likely due to the loss of the C-terminal RG/RGG region (fig. S4C). To investigate FMRP incorporation into FUS condensates, we added ${ }^{\text {Alexa647 }}{ }^{F M R P} P_{L C R}$ to preformed FUS droplets in the presence or absence of sc1 RNA. FMRP was equally enriched in FUS ${ }^{\mathrm{WT}}$, FUS ${ }^{\mathrm{P} 525 \mathrm{~L}}$, and $\mathrm{FUS}^{\Delta 14}$ condensates in the absence of scl RNA, and scl RNA increased the enrichment of ${ }^{\text {Alexa647}}{ }^{F M R P} P_{\text {LCR }}$ in these condensates for all FUS proteins $\left({ }^{\text {Alexa647 }}{ }^{\text {FMRP }}\right.$ LCR enrichment ratio in $\mathrm{FUS}^{\mathrm{WT}}=1.5 \pm 0.04, \mathrm{FUS}^{\mathrm{WT}}+$ $\mathrm{scl}=4.0 \pm 0.05$ FUS $^{\mathrm{P} 525 \mathrm{~L}}=1.3 \pm 0.004$, FUS $^{\mathrm{P} 525 \mathrm{~L}}+\mathrm{scl}=3.5 \pm 0.17$, $\mathrm{FUS}^{\Delta 14}=1.4 \pm 0.008$, and FUS ${ }^{\Delta 14}+\mathrm{scl}=2.7 \pm 0.04 ;{ }^{* * *} P<0.001$ and ${ }^{* * *} P<0.0001$; Fig. $2, \mathrm{C}$ and D). These results show that FMRP partitions into FUS droplets via protein-protein and protein-RNA interactions. The finding that wild-type and mutant FUS do not 
A
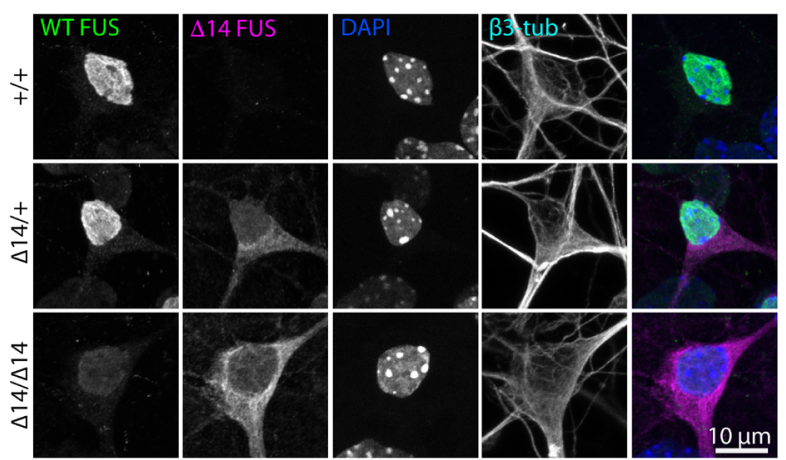

C

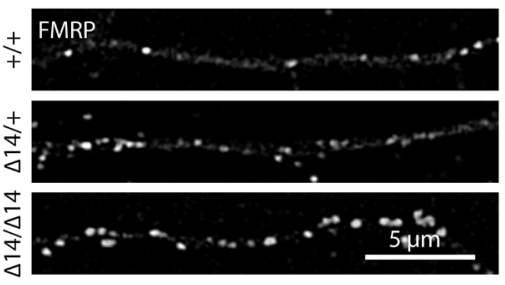

D

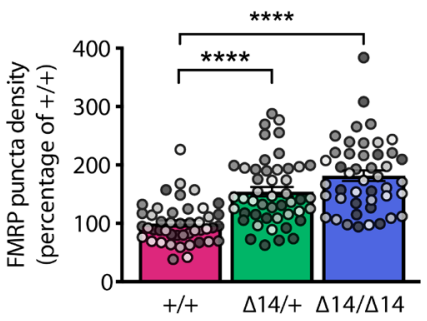

E

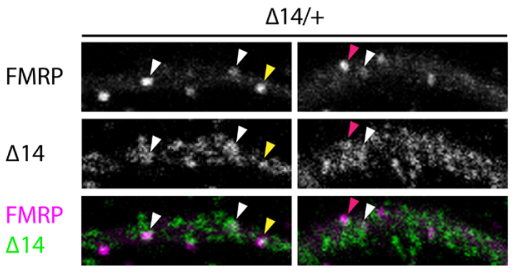

$\Delta 14 / \Delta 14$

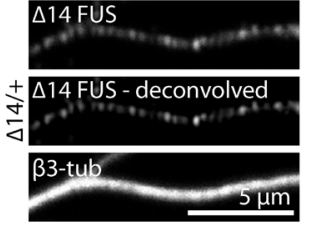

$\mathbf{F}$
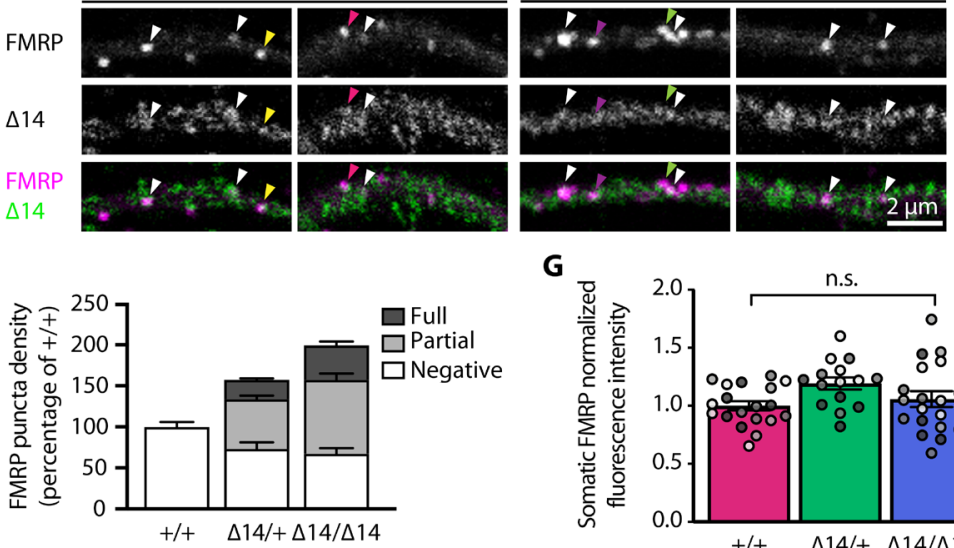

G

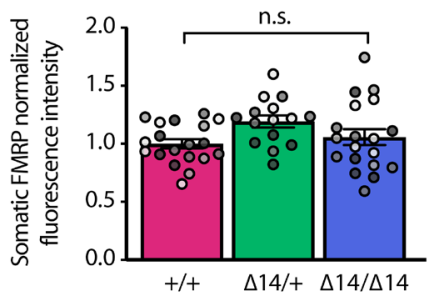

H

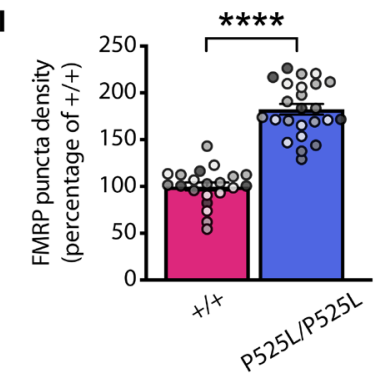

Fig. 1. FUS mutants induce increased FMRP puncta density in MN axons. (A) Representative images of primary MNs at 5 days in vitro (DIV 5). Wild-type (WT) FUS (green), detected with a C-terminal antibody, is primarily localized in the nucleus in Fus ${ }^{+/+}$and Fus ${ }^{\Delta 14 /+}$ neurons. $\Delta 14$ FUS (magenta) is enriched in the cytoplasm of Fus ${ }^{\Delta 14 /+}$ and $\mathrm{Fus}^{\Delta 14 / \Delta 14}$ neurons. Nuclei are labeled with 4',6-diamidino-2-phenylindole (DAPI) (blue), and $\beta 3$-tubulin (cyan) is used as a neuronal marker. Low-intensity wild-type FUS-positive nuclear staining in Fus ${ }^{\Delta 14 / \Delta 14}$ neurons is due to antibody cross-reactivity with another FET protein, likely EWSR1. (B) $\Delta 14$ FUS distribution in a Fus ${ }^{\Delta 14 /+}$ MN axon. $\triangle 14$ FUS signal detected by confocal microscopy (top) and the deconvoluted signal (middle). Neurons were grown in microfluidic devices, and $\beta 3$-tubulin is used to identify axons. (C) Representative deconvolved images of FMRP axonal puncta in Fus ${ }^{+/+}, F^{4} s^{\Delta 14 /+}$, and Fus ${ }^{\Delta 14 / \Delta 14}$ MNs grown in microfluidic chambers (MFCs) (DIV 8). (D) Quantification of axonal FMRP puncta density in Fus ${ }^{+/+}$, Fus $^{\Delta 14 /+}$, and Fus ${ }^{\Delta 14 / \Delta 14}$ MNs $(n=4$, axons $=45$ to $47, * * * * P<0.0001$; Kruskal-Wallis, followed by Dunn's post hoc test). (E) Representative images showing axonal FMRP puncta either fully (white arrowheads) or partially (colored arrowheads) positive for $\Delta 14 \mathrm{FUS}$ in Fus ${ }^{\Delta 14 /+}$ and Fus ${ }^{\Delta 14 / \Delta 14}$ MNs. (F) Segmentation of FMRP puncta density into fully $\Delta 14$ positive and partially $\Delta 14$ positive and negative. (G) Quantification of somatic FMRP fluorescence in Fus ${ }^{+/+}$, Fus ${ }^{\Delta 14 /+}$, and Fus ${ }^{\Delta 14 / \Delta 14}$ HB9.::GFP (green fluorescent protein)-positive MNs $\left(n=4, \mathrm{MNs}=15\right.$ to 19). (H) Representative images of FMRP axonal puncta in FUS ${ }^{+/+}$and FUS ${ }^{P 525 / P 525 L}$ iPSCderived MNs grown in MFCs. (I) Quantification of axonal FMRP puncta density in FUS $S^{+/+}$and FUS $S^{P 525 L / P 525 L}$ iPSC-derived MNs as shown in (F) $(n=4$, axons $=21$ to 24 ; ${ }^{* * * *} P<0.0001$, Student's $t$ test). Independent experiments are visualized in different shades of gray in the graphs. n.s., not significant. 
A
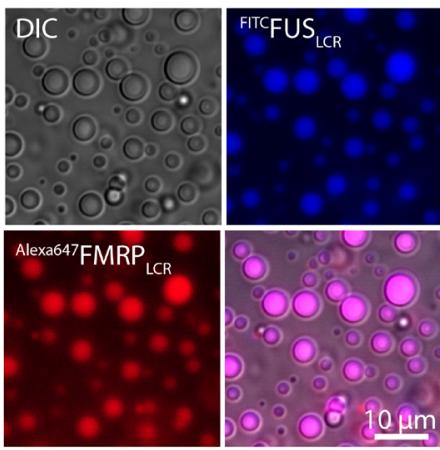

C

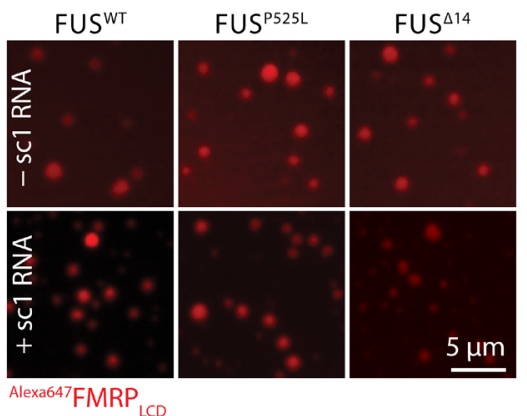

B

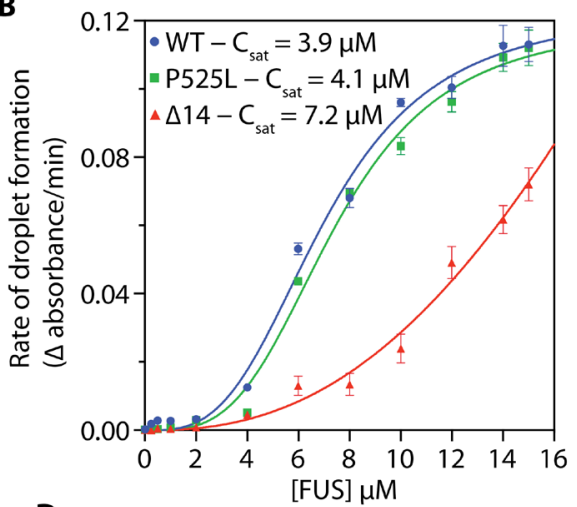

D

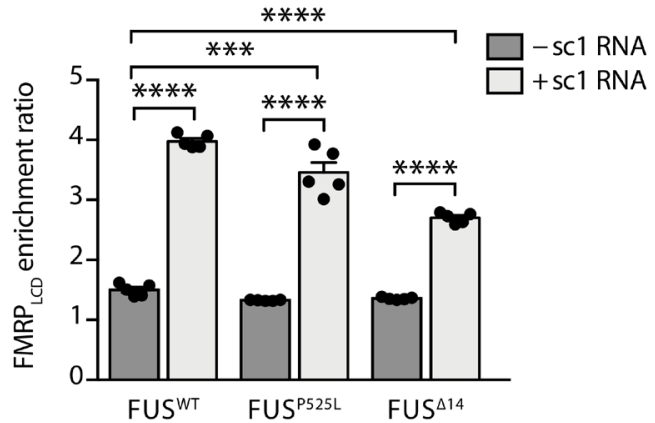

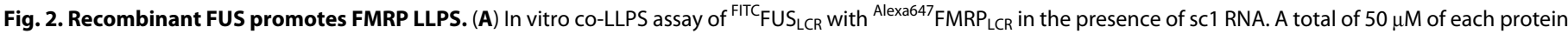

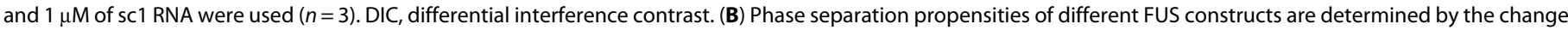

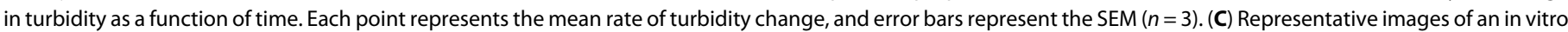

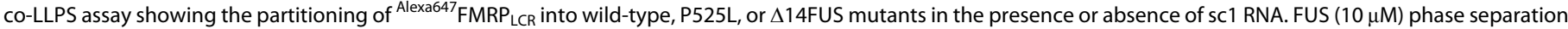

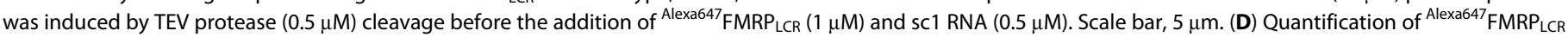

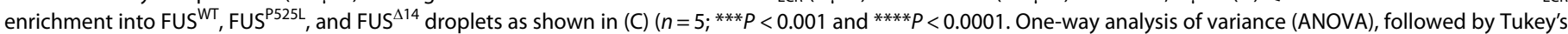
multiple comparisons test).

significantly differ is not unexpected as wild-type FUS is physiologically prevalently nuclear, while these assays recapitulate the behavior of the proteins when present in the same compartment.

To further analyze FUS-induced FMRP sequestration in a cellular environment, we took advantage of the ability of mutant FUS to generate intracellular condensates upon overexpression (7). Overexpression of either NLS-lacking FUS $\left({ }^{\text {mCherry }} \mathrm{FUS}^{513 \mathrm{x}}\right)$ or mutant FUS ( ${ }^{\mathrm{Flag}}{ }_{\mathrm{FUS}}{ }^{\mathrm{P} 25 \mathrm{~L}}$ ) in HeLa cells induced the formation of large, cytoplasmic FUS-positive condensates (fig. S5A, left), which led to the sequestration of endogenous FMRP (fig. S5A). We found that brief ( $\sim 18$-hour) overexpression of ${ }^{\text {Cherry }^{2}}$ FUS $^{513 x}$ led to the presence of small FMRP puncta decorating larger FUS condensates (fig. S5A, top), as well as condensates with a more homogeneous distribution of FMRP and FUS (fig. S5A, middle), possibly reflecting different phases of incorporation or a multiphasic behavior reminiscent of FMRP-Caprin 1 condensates (24). Overexpression of ${ }^{\text {Flag }}{ }_{\text {FUS }}{ }^{\text {P525L }}$ in primary MNs also led to the formation of distinct FUS condensates that were positive for endogenous FMRP (fig. S5; arrowheads indicate FMRP-positive FUS puncta).

Because FUS overexpression may induce the formation of stress granules, the increase in FMRP condensates could be due to cellular stress rather than a direct consequence of cytoplasmic mutant FUS. To address this, we generated mouse embryonic fibroblasts (MEFs) from our $\Delta 14$ FUS mouse model, which endogenously expresses mutant FUS. When we looked at endogenous FMRP, we found that the presence of mutant FUS led to a dose-dependent increase in the number of cells with spontaneous FMRP condensates $\left(>0.5 \mu \mathrm{m}^{2}\right)$ compared to controls (fig. S5, C to F; percentage of MEFs with FMRP puncta: $\mathrm{Fus}^{+/+}=46.2 \pm 2.1 \%$, Fus ${ }^{\Delta 14 /+}=55.8 \pm 3.6 \%$, and Fus $^{\Delta 14 / \Delta 14}=63.8 \pm 5.4 \%$; $\left.{ }^{* *} P<0.05\right)$. The large majority of these puncta were negative for stress granule markers, such as Ras-GAP SH3 domain binding protein 1 (G3BP1) (fig. S5, C and D; percentage of MEFs with G3BP1 puncta Fus ${ }^{+/+}=1.5 \pm 0.6 \%$, Fus $^{\Delta 14 /+}=0.8 \pm 0.4 \%$, Fus $\left.^{\Delta 14 / \Delta 14}=1.7 \pm 0.7 \%\right)$. Together, these findings further indicate that cytoplasmic mutant FUS increases localization of FMRP in cytoplasmic FUS condensates in neuronal and non-neuronal cells and provides evidence that the presence of FMRP in these structures is not a secondary effect triggered by cellular stress.

\section{FUS and FMRP repress translation in vitro}

Biomolecular condensates are thought to antagonize translation $(25,26)$, and FMRP phase separation correlates with translation inhibition (23). To test whether FUS condensates can directly affect protein synthesis, we took advantage of an in vitro translation assay in which recombinant MBP-FUS fusion proteins were directly added to a commercial rabbit reticulocyte cytoplasmic extract and its phase separation was induced by TEV protease cleavage. Quantification of the bioluminescence of the firefly luciferase, as a reporter for the translation of its mRNA, showed that all FUS proteins significantly suppressed translation, and addition of $\mathrm{FMRP}_{\mathrm{LCR}}$ induced a further 
decrease in luciferase translation (normalized bioluminescence per minute in buffer control $=1 \pm 0.12$, buffer $+\mathrm{FMRP}=0.2 \pm 0.02$, $\mathrm{FUS}^{\mathrm{WT}}=0.27 \pm 0.05, \mathrm{FUS}^{\mathrm{WT}}+\mathrm{FMRP}=0.17 \pm 0.02, \mathrm{FUS}^{\mathrm{P} 525 \mathrm{~L}}=$ $0.27 \pm 0.03, \mathrm{FUS}^{\mathrm{P} 525 \mathrm{~L}}+\mathrm{FMRP}=0.08 \pm 0.01, \mathrm{FUS}^{\Delta 14}=0.15 \pm 0.01$, $\mathrm{FUS}^{\Delta 14}+\mathrm{FMRP}=0.08 \pm 0.01 ;{ }^{* * *} P<0.0001$; Fig. $\left.3 \mathrm{~A}\right)$. These results support FUS having a repressive role on protein synthesis and that its mislocalization to the cytoplasm and not the mutations per se is key to driving this gain-of-function mechanism. FMRP has an additive, although not significant, effect on translation inhibition.

\section{Cytoplasmic FUS represses translation in MNs}

Two reports have recently shown that mutant FUS overexpression can impair protein synthesis in neurons $(7,27)$, but whether this also occurs at physiological FUS expression is not currently known. We analyzed de novo protein synthesis in primary motoneuronal cultures, using the methionine analog L-azidohomoalanine [(AHA) $2 \mathrm{mM}, 30$-min incubation] in combination with click chemistry. We found that, compared to wild-type controls, AHA labeling was reduced by $13 \%$ in $\mathrm{Fus}^{\Delta 14 /+}$ and by $20 \%$ in $\mathrm{Fus}{ }^{\Delta 14 / \Delta 14}$ primary MNs (average AHA intensity in Fus ${ }^{++}$MNs $100 \pm 3.8$, Fus $^{\Delta 14 /+}$ MNs $87.4 \pm 4.5$, and $F u s^{\Delta 14 / \Delta 14}$ MNs $77.7 \pm 2.7 ;{ }^{*} P<0.05$ and ${ }^{* * * *} P<$ 0.0001 ; Fig. $3, \mathrm{~B}$ and C). As a positive control, we showed that pretreatment with the translation inhibitor anisomycin $(40 \mu \mathrm{M}, 20 \mathrm{~min})$ reduced the AHA signal by $80 \%$, indicating the specificity of the labeling (fig. S6, B and C). In contrast, in FUS knockout MNs, no significant effect on de novo protein synthesis was detected (average AHA intensity in $\mathrm{Fus}^{+++} \mathrm{MNs} 100 \pm 5.5, \mathrm{Fus}^{+/-} \mathrm{MNs}$ $115 \pm 7.7$, and $\mathrm{Fus}^{-/-}$MNs $114 \pm 6.0$; Fig. 3D and fig. S6A), indicating that inhibition of translation is due to a gain of function of mutant FUS.

To test whether this deficit was conserved in human models of FUS-ALS, we performed the same assay in iPSC-derived MNs carrying the common ALS-associated NLS mutation P525L and compared them to isogenic controls. Similarly to mouse MNs, AHA labeling was decreased by $20 \%$ in mutant FUS human MNs (isogenic control $100 \pm 3.1$ and $F U S^{P 525 L / P 525 L} 79.4 \pm 2.2$; $^{* * * *} P<0.0001$; Fig. $3, \mathrm{E}$ and F), underlining that translation inhibition stems from FUS cytoplasmic mislocalization and is not mutation specific.

\section{Mutant FUS does not alter translation by association with polysomes}

Despite its low cytoplasmic levels, wild-type FUS binds proteins of both the small and large ribosomal subunits (28), suggesting that it may interact with assembled ribosomes. Given the increase in cytoplasmic levels of mutant FUS, we asked whether association with ribosomes could account for the observed changes in translation. To investigate this, we performed polysome cosedimentation assays, where separation of the heavier polysomal fractions from monosomes (80S), the individual ribosomal subunits ( $60 S$ and $40 S$ ), and the lighter free cytosolic complexes (fig. S7Ai) allows the analysis of the association of specific proteins with the translation components. As expected, most of wild-type FUS cosedimented with free cytosolic complexes, but significant levels also cosedimented with polysomes in both $\mathrm{Fus}^{+/+}$and Fus ${ }^{\Delta 14 /+}$ samples (fig. S7Aii). However, $\Delta 14$ FUS did not cosediment with polysomal fractions, despite its large cytoplasmic localization (fractions 8 to 11; fig. S7Aii), and was only present in the lighter part of the gradient, which contains subpolysomal components, in Fus ${ }^{\Delta 14 /+}$ and Fus ${ }^{\Delta 14 / \Delta 14}$ samples (fractions 1 to 6; fig. S7Aii).
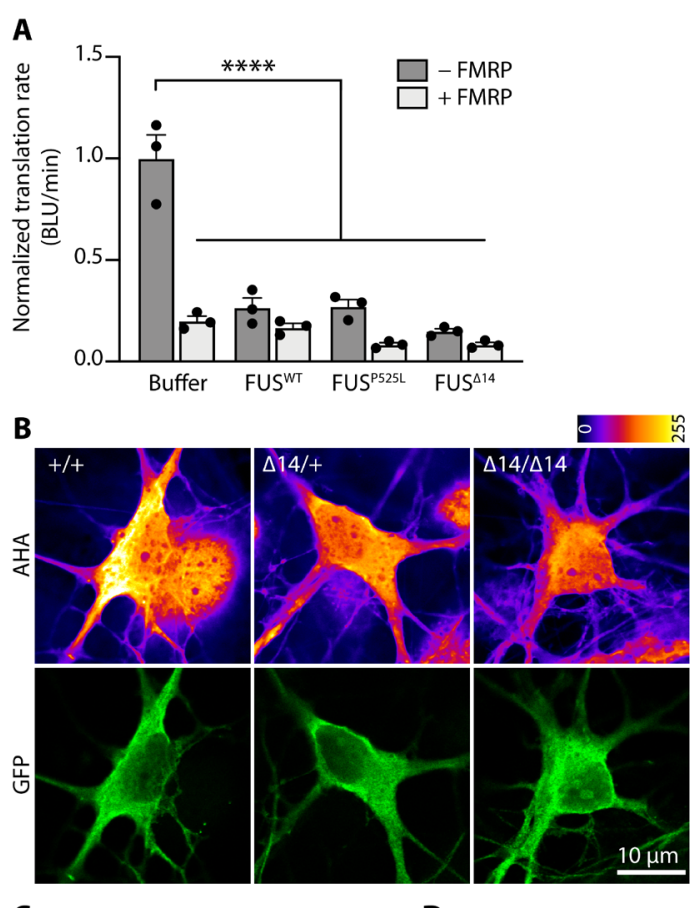
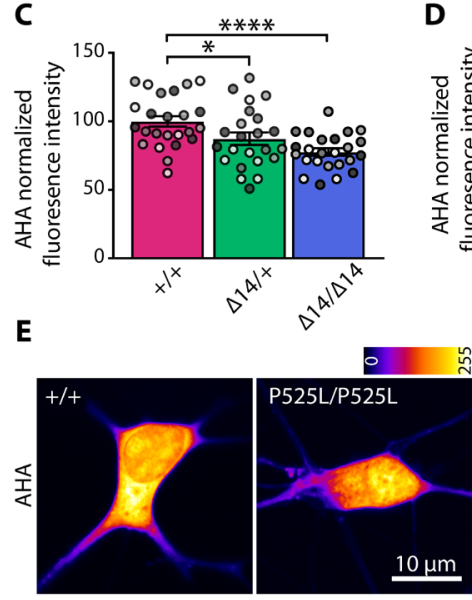

D

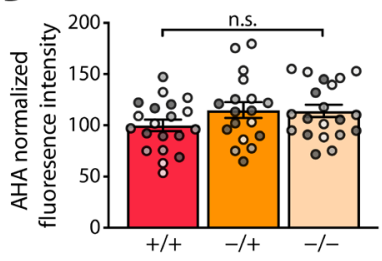

$\mathbf{F}$

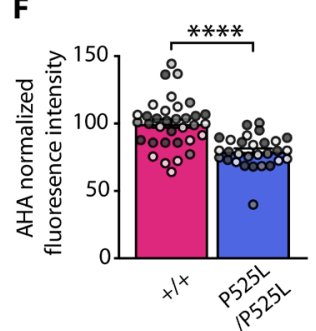

Fig. 3. Mutant FUS represses translation in vitro and in cultured MNs. (A) Recombinant FUS ${ }^{\mathrm{WT}}$, FUS ${ }^{\mathrm{P} 25 \mathrm{~L}}$, and FUS $\mathrm{F}^{\Delta 14}(10 \mu \mathrm{M})$ phase separation was induced by TEV protease $(0.5 \mu \mathrm{M})$ cleavage. Proteins were added to an in vitro rabbit reticulocyte translation system with luciferase mRNA in the absence or presence of FMRP $(10 \mu \mathrm{M})$. Change in bioluminescence (BLU) rate is used as a reporter for translational activity. All results were normalized to buffer control (+TEV) $\left(n=3,{ }^{* * * *} P<0.0001\right.$; one-way ANOVA, followed by Sidak's multiple comparisons test). (B) Representative images of primary Fus ${ }^{+/+}$, Fus ${ }^{\Delta 14 /+}$, and Fus ${ }^{\Delta 14 / \Delta 14}$ MNs metabolically labeled using the methionine analog AHA ( $2 \mathrm{mM}, 30 \mathrm{~min}$ ) and click chemistry. AHA labeling is visualized using the LUT fire (top), and MNs are identified by GFP expression under the HB9 promoter (bottom). (C) Quantification of the AHA labeling as shown in (C). Mean fluorescence intensity values are normalized to $\mathrm{Fus}^{+/+}(n=4, \mathrm{MNs}=27$ to 28; oneway ANOVA, followed by Dunnett's multiple comparisons test, ${ }^{*} P<0.05$ and $\left.{ }^{* * * *} P<0.0001\right)$. (D) Quantification of translation assays carried out in $\mathrm{Fus}^{+/+}, \mathrm{Fus}^{+/-}$, and $\mathrm{Fus}^{-/-}$MNs. Mean fluorescence intensity values are normalized to $\mathrm{Fus}^{+/+}$ $(n=3, \mathrm{MNs}=18$ to 20$)$. (E) Representative images of isogenic control $\left(F U S^{+/+}\right)$and FUS $^{\text {P525L/P525L }}$ iPSC-derived MNs metabolically labeled with AHA (2 mM, $30 \mathrm{~min}$ ). (F) Quantification of the effect in (E). Fluorescence values are normalized to $\mathrm{FUS}^{+/+}$ MNs ( $n=3, \mathrm{MNs}=29$ to 34 ; Student's $t$ test, $\left.{ }^{* * * *} P<0.0001\right)$. Independent experiments are visualized in different shades of gray in the graphs. 
Because both FMRP and SMN are also known to associate with the translational machinery $(29,30)$, we examined their cosedimentation profile in the $\triangle 14$ FUS model. While both FMRP and SMN interact with $\Delta 14$ FUS (fig. S2A), when analyzing their cosedimentation, we found that the distribution of SMN was unaltered by the expression of mutant FUS, whereas FMRP was depleted from the polysomal fractions in both $F u s^{\Delta 14 /+}$ and Fus ${ }^{\Delta 14 / \Delta 14}$ samples (fig. S7A). We confirmed the weaker association of FMRP with ribosomes in $\triangle 14$ FUS MNs using proximity ligation assays (PLAs) between FMRP and the ribosomal protein RPL26 (normalized FMRP-RPL26 (ribosomal protein L26) PLA puncta in $\mathrm{Fus}^{+/+}=1.0 \pm 0.14, \mathrm{Fus}^{\Delta 14 /+}=0.18 \pm 0.04$, and $F u s^{\Delta 14 / \Delta 14}=0.37 \pm 0.1 ;{ }^{*} P<0.05$ and ${ }^{* * *} P<0.0001$; fig. S7, C and D). We found FUS depletion $\left(\mathrm{Fus}^{-/}\right)$to have a similar effect on FMRP-ribosome association both in cosedimentation assays (fig. S7B) and in FMRP-RPL26 PLAs (normalized FMRP-RPL26 PLA puncta in $\mathrm{Fus}^{+/+}=1.0 \pm 0.12, \mathrm{Fus}^{+/-}=0.9 \pm 0.21$, and $\mathrm{Fus}^{-/-}=0.44 \pm 0.08$; ${ }^{*} P<0.001$; fig. S7E).

In summary, these results show that FUS mutations impair its association with polysomes and that wild-type FUS promotes the association of FMRP with the translation machinery. Given that the global level of protein synthesis is not affected in FUS knockout MNs and that polysomal localization of FUS and FMRP is impaired in both knockout and mutant FUS neurons, we conclude that these alterations cannot be the main driver for the translation phenotype observed in Fig. 3, B to F.

\section{Mutant FUS impairs translation of FMRP target RNAs in vivo}

We next investigated whether mutant FUS expression could affect the translation of RNAs specifically bound to either FUS or FMRP in vivo. We crossed $\Delta 14$ FUS mutant and FUS knockout lines with mice expressing both the Cre-dependent hemagglutinin (HA)-tagged RPL22 ribosomal subunit (Rpl22 ${ }^{H A}$, RiboTag) and a MN-specific Cre-recombinase (Chat-Cre) (31). We obtained two triple transgenic lines, Chat-Cre/Fus ${ }^{\Delta 14 / \Delta 14} /$ Rpl$_{2} 2^{H A}$ and Chat-Cre/Fus ${ }^{-/-} / \mathrm{Rpl}_{2} 2^{\mathrm{HA}}$, allowing us to immunopurify ribosome-bound transcripts from $\mathrm{MNs}$ within the mouse spinal cord. We refer to ribosome-associated mRNAs (Ribo) as the "translatome" throughout the manuscript (Fig. 4A and fig. S8, A to C). To validate MN specificity of purified RNAs, we performed quantitative polymerase chain reaction (qPCR) experiments that confirmed enrichment for the motor neuronal genes Chat and $R p l 22^{H A}$ and depletion of glial gene Pmp22 (Fig. 4B).

To identify changes in the translatome caused by the cytoplasmic mislocalization of mutant FUS, we compared changes of RiboFus $^{\Delta 14 / \Delta 14}$ versus littermate controls to ones in Ribo-Fus ${ }^{-/-}$versus their own littermate controls. We found 21 up-regulated and 26 downregulated transcripts (adjusted $P<0.1$ ) when comparing the RiboFus $^{\Delta 14 / \Delta 14}$ translatome to wild-type littermate controls (Fig. $4 \mathrm{C}$ and table S1), while the analysis of Ribo-Fus ${ }^{-/}$and wild-type littermate control (fig. S8D and table S1) identified 8 up-regulated and 39 downregulated transcripts (adjusted $P<0.1$ ). The comparison of the $z$ score for each gene in the Ribo-Fus ${ }^{\Delta 14 / \Delta 14}$ and Ribo-Fus ${ }^{-/-}$experiments showed little correlation between the two datasets (Pearson correlation coefficient $\left.r=0.28, P<2.2 \times 10^{-16}\right)$. Only 3 transcripts were significantly differentially represented in both Ribo-Fus ${ }^{\Delta 14 / \Delta 14}$ and Ribo-Fus ${ }^{-/}$, while others were specific to the translatome of each genotype ( 3 transcripts with an adjusted $P<0.1$ in both RiboFus $^{\Delta 14 / \Delta 14}$ and Ribo-Fus ${ }^{-7-}, 43$ transcripts only in Ribo-Fus ${ }^{\Delta 14 / \Delta 14}$, and 39 transcripts only in Ribo-Fus ${ }^{-/-}$; Fig. 4D and table S2). One of the commonly altered transcripts was Taf15, a member of the FET family (along with Fus and Ewsr1), likely as a compensatory response to FUS nuclear loss. We performed gene ontology (GO) term analysis on Ribo-Fus ${ }^{\Delta 14 / \Delta 14}$ and Ribo-Fus ${ }^{-/-}$(fig. S8). Mutant FUS caused an up-regulation of genes related to ribosomal biogenesis, which was absent in FUS knockout.

We next asked whether RNAs bound by FUS or by FMRP were affected at the translatome level. We used published and widely used CLIP (individual-nucleotide resolution Cross-Linking and ImmunoPrecipitation) data to select RBP-bound RNAs $(30,32)$. Because FUS, unlike FMRP, mostly binds pre-mRNA intronic sequences, we selected only transcripts where FUS binds within the mature RNA sequence [5' untranslated region (5'UTR), CDS (coding sequence), and $3^{\prime} \mathrm{UTR}$ ]. We plotted the distribution of target RNAs (bound by a defined RBP) and non-target RNAs (not bound) (fig. S9B) as a cumulative frequency of their $z$ scores and used non-target RNAs with similar expression levels to target RNAs to avoid a bias deriving from gene expression levels (fig. S9 for FUS and fig. S10 for FMRP). In this analysis, a shift of the cumulative frequency curve toward the right indicates an up-regulation of targets in the condition of interest, and a leftward shift indicates a down-regulation $(33,34)$. The comparison between FUS targets versus non-target controls shows a minor, albeit significant, right shift (up-regulation) of the cumulative distribution in Ribo-Fus ${ }^{\Delta 14 / \Delta 14}$ (distance $D=0.05 ; P=1.20 \times 10^{-5}$ ), while no change was detected in the Ribo-Fus ${ }^{-/}$dataset $(D=0.02 ; P=0.26$; fig. S9C). Conversely, when investigating FMRP targets, we found more widespread changes (Fig. 4, E and F, and fig. S10B). We found that FMRP target distribution was down-regulated selectively in Ribo-Fus ${ }^{\Delta 14 / \Delta 14}$ (left shift, $D=0.16, P=3.10 \times 10^{-15}$; Fig. $4 \mathrm{G}$ ), while no distribution change was present in Ribo-Fus ${ }^{-/-}(D=0.04, P=0.13$; Fig. $4 \mathrm{H})$. This indicates that the presence of mutant FUS in the cytoplasm leads to impaired ribosome association of FMRP targets, supporting the translation inhibition by FUS and FMRP condensates shown in previous assays (Figs. 1 to 3 ).

GO analysis of down-regulated FMRP targets highlighted terms related to axonal compartments in Ribo-Fus ${ }^{\Delta 14 / \Delta 14}$, while these were absent in Ribo-Fus ${ }^{-1-}$ (fig. S10C; Ribo-Fus ${ }^{\Delta 14 / \Delta 14}$ : fold change $<0, P<0.05,33$ genes; and fig. S10D; Ribo-Fus ${ }^{-1-}$ : fold change $<0$, $P<0.05,21$ genes). We then compared the RiboTag to published mass spectrometry on iPSC-derived FUS P525L MNs used throughout this work (35). We found seven human orthologs, with FMRP binding sites at the transcript level, to be down-regulated $(z$ score $>1$ in both datasets) in both Ribo-Fus ${ }^{\Delta 14 / \Delta 14}$ and FUS ${ }^{P 525 L / P 525 L} \mathrm{MN}$ mass spectrometry (respective mouse genes: Dnm1, Usp9x, Trim2, Pld3, Foxk2, Arfgef1, and Tmod2; table S3). TRIM2 encodes a ubiquitin ligase that targets the large neurofilament, and TRIM2 recessive loss-of-function mutations have been found to be causative for hereditary motor axonal neuropathy (36).

\section{DISCUSSION}

Cytoplasmic mislocalization and nuclear depletion of FUS are hallmarks of FUS-ALS, and the degree of cytoplasmic misplacement induced by different disease-causing mutations correlates with disease severity (3). The molecular mechanisms underlying FUSmediated neuronal toxicity are unclear; however, alterations in phase separation propensities and protein translation have been proposed to have a relevant role $(7,27,37,38)$. Moreover, FUS expression levels, similarly to many RBPs, are physiologically highly regulated, and both exogenous overexpression and complete depletion were found 
A
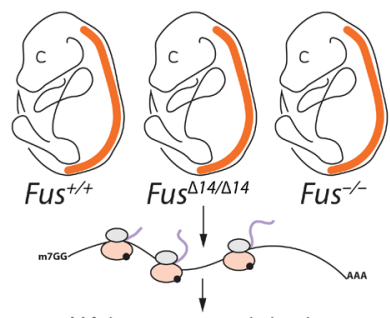

HA immunoprecipitation $\downarrow$

Ribosome-bound mRNA isolation and sequencing

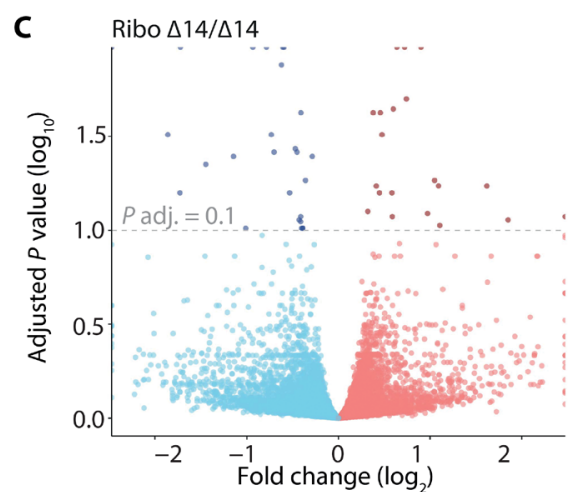

E Ribo $\Delta 14 / \Delta 14$, FMRP targets

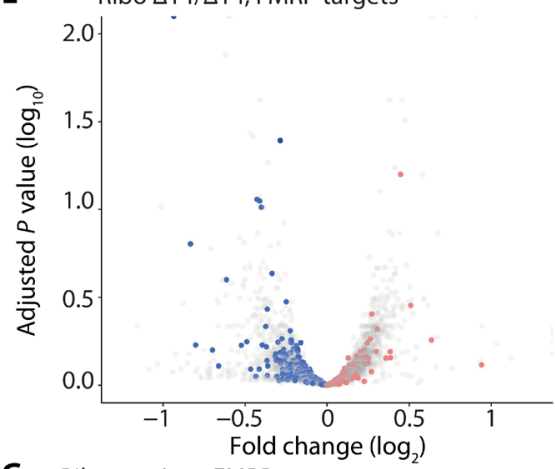

G Ribo $\Delta 14 / \Delta 14$ FMRP targets, $z$ score

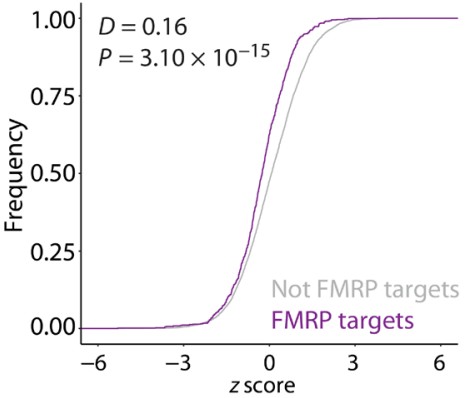

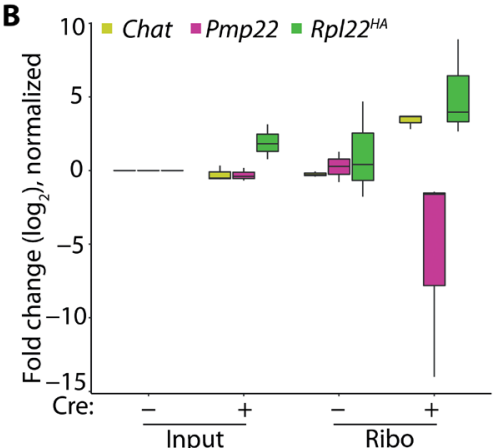

D Adj. $P<0.1 \triangle 14 / \Delta 14$ only $\bullet-/-$ only $\bullet$ both

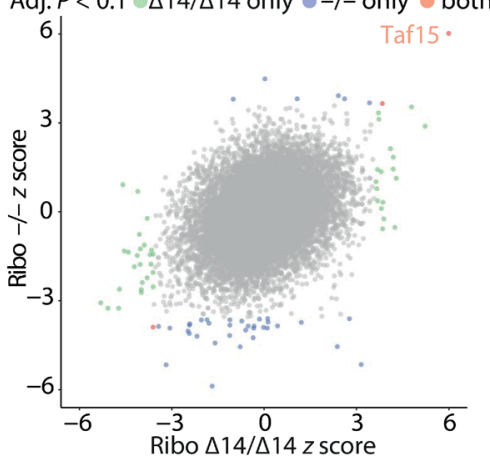

$\mathbf{F}$

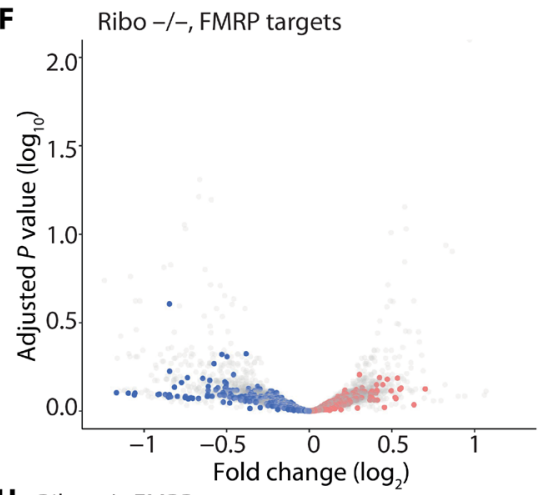

H Ribo-/-FMRP targets, $z$ score

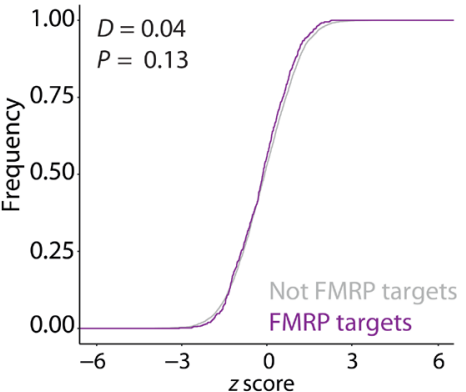

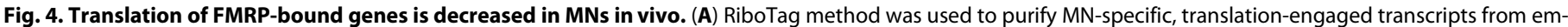

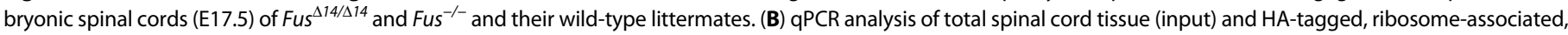

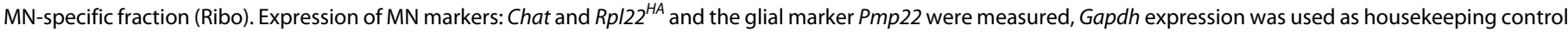

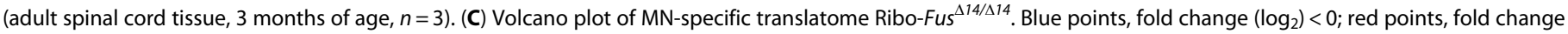

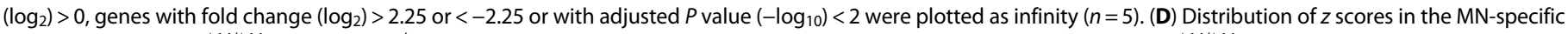

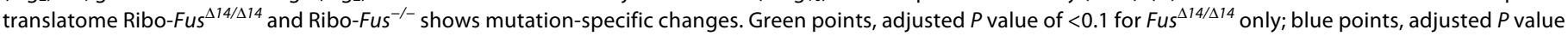

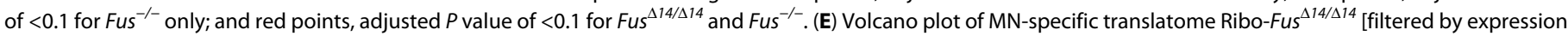

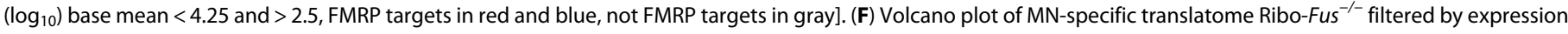

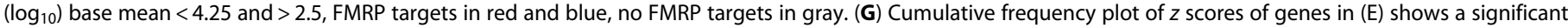

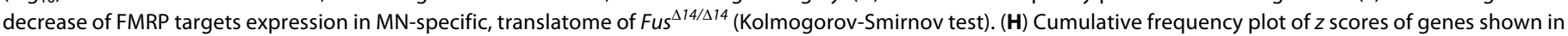
(F) shows no change of FMRP targets expression in MN-specific, translatome of Fus ${ }^{-/-}$(Kolmogorov-Smirnov test). 
to affect numerous cellular processes, causing neurodegeneration and lethality $(4,39-41)$. We investigated the consequences of mutant FUS mislocalization using both mouse and human models, where FUS mutants are expressed at endogenous levels. We analyzed neurons that express mutant FUS both in heterozygosity, as in patients with ALS, and in homozygosity, to better uncover phenotypes. We found that FUS forms condensates where FMRP, another RBP strongly linked to translation regulation and neurodegeneration (42-45), is sequestered. We show that endogenous expression of FUS mutants is sufficient to impair translation both in mouse and human MNs. Last, we demonstrate that translation of FMRP target RNAs is impaired in vivo in MNs, establishing a pathogenesis paradigm by which mutant FUS impairs translation of specific sets of transcripts by altering the phase behavior of another RBP (Fig. 5).

Recently, numerous studies have demonstrated that FUS can undergo LLPS and that cytoplasmic FUS granules are biomolecular condensates $(7,11)$. Cytoplasmic condensates have been shown to be involved in various aspects of RNA metabolism. Not only phase separation of specific RBPs is required for the formation of transport granules and stress granules but also the fine tuning of condensate assembly and disassembly is key to controlling RNA availability and, ultimately, translation. In agreement with this, activity-dependent protein translation has been associated with the disassembly of RNA granules and the consequent release of RBP-bound transcripts. Neuronal activity triggers $\beta$-actin mRNA granule disassembly $(46,47)$, associated with activity-dependent protein synthesis; similarly, injury-induced disassembly of G3BP1 granules results in the translation of G3BP1-bound RNAs (26).

Mutant $\triangle 14$ FUS condensates are present throughout the MN cell body and neurites (Fig. 1, A and B) and are likely to have an impact on RBP dynamics and RNA metabolism before the formation of pathological aggregates seen in post mortem tissue (5). The composition of biomolecular condensates is heterogeneous, and the proteins that partition in these structures dictate their biological function. While LCRs of proteins are required for LLPS, RBPs containing these regions have different propensities to phase-separate, and in vitro studies have demonstrated that FUS is particularly prone to undergo this process. Moreover, FUS phase separation can favor the copartitioning of so-called "client proteins" that contain LCRs but would not normally form condensates at physiological concentrations (48). FUS condensates could, therefore, act as a scaffolding in which other proteins partition, and the resulting aberrant compartmentalization of these factors could determine alterations in their biological activity. With this in mind, we questioned whether the presence of cytoplasmic FUS condensates could alter the distribution and partitioning of a wider RBP network. Because some FUS-interacting RBPs are also found in FUS inclusions in post mortem tissue or are disrupted in models of FUS-ALS $(4,15,49)$, we decided to analyze the distribution of the two most well-characterized RBPs: FMRP and SMN. When we analyzed the distribution of axonal FMRP, we found a dose-dependent increase in FMRP condensates in mutant FUS primary MNs (Fig. 1, C, D, H, and I) and iPSCderived MNs, which is not due to alterations in FMRP expression levels but likely reflects a shift in the equilibrium between diffuse FMRP and FMRP granules. We investigated the axonal distribution of these RBPs because they have key roles in RNA transport and axonal function; however, it is likely that the effect is not restricted to this compartment, and we found alterations in the frequency of FMRP condensates also in non-neuronal MEF cells (fig. S5, C and D). Although FMRP granules can be induced by cellular stress, FUSinduced FMRP condensates are negative for stress granule markers (fig. S5, C and D). We therefore propose that the increased FMRP condensate formation is due to a cytoplasmic aberrant function of mutant FUS. Although other cytoplasmic RBPs containing LCRs are likely to copartition within FUS condensates, we did not find any alteration in the distribution of SMN (fig. S2, K and L). Although the expression of mutant FUS may still affect SMN functionality [for example at the nuclear level, as previously reported (4)], this indicates that, in addition to protein-protein interaction, other factors are required to promote FUS-induced condensate formation.

We report that expression of FUS mutants at endogenous levels impairs translation, both in physiological models of disease (heterozygous FUS mutant mice) and in MNs carrying homozygous mutations. A recent study, where FUS was overexpressed in human

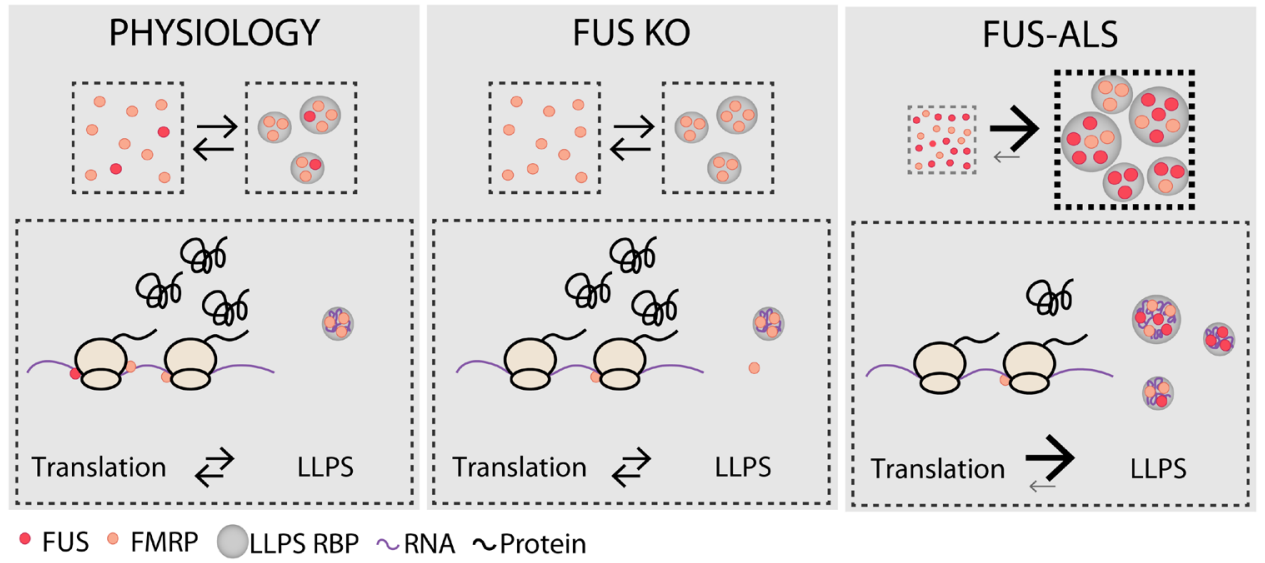

Fig. 5. Proposed model of mutant FUS cytoplasmic gain of function. Under control conditions (left), low levels of FUS are present in the cytoplasm and the phase separation of FUS and FMRP are at a physiological equilibrium. Loss of FUS (middle) results in a reduction of FMRP association with the translational machinery; however, this does not induce significant alterations in FMRP LLPS or global protein translation. In FUS-ALS (right), the increased cytoplasmic localization of FUS shifts the LLPS equilibrium of both FUS and FMRP, resulting in an increase in cytoplasmic condensates. This is associated with a depletion of the proteins from the translational machinery and an overall decrease in protein synthesis. 
embryonic kidney (HEK) 293 cells, has shown that FUS-mediated translation inhibition was associated with increased cosedimentation of FUS mutants with polysomes (38). In our model, however, we find that endogenously expressed mutant FUS does not associate with the translational machinery using biochemical and imaging approaches, a difference that could be due to the overexpression of the protein or to an alternative regulation in HEK293 cells. While FUS-mediated translation regulation has not yet been studied in detail, the role of FMRP as a translation repressor is well established. FMRP inhibits translation through several mechanisms, including polysome binding, miRNA and RNA-induced silencing complexdependent repression, or binding to translation initiation factors (43). More recently, FMRP LLPS has been shown to correlate with translation inhibition in vitro (23), and in vivo FMRP granule formation decreases the translation of FMRP-bound synaptic targets (50). This adds complexity to the FMRP-dependent regulation of translation and supports a model in which an increase in FMRP condensates could be associated with decreased translation of FMRP targets. To explore how the translation landscape is affected by mutant FUS expression in vivo, we sequenced ribosome-engaged transcripts from MNs. FUS mutations induce transcript expression changes, and we have previously shown that these are comparable to but weaker than FUS knockout $(4,51)$. However, when we compared the translatome datasets, we found specific changes in the $\Delta 14$ FUS compared to the knockout translatome (Fig. 4D), demonstrating that mutant FUS affects the translatome through a gain-offunction mechanism. When we analyzed FMRP target RNAs, we found a selective depletion of these transcripts from the purified ribosomal fractions of our mutant $\Delta 14$ FUS model, with no changes in FUS knockout (Fig. 4G). The depletion induced by mutant FUS is notably similar to that described in an FMRP knockout model [fig. S10C; reanalyzed from (34); Ribo-Fmr1 ${ }^{-/ y} D=0.18, P=2.56 \times$ $10^{-11}$; total RNA Fmr1 $\left.1^{-/ y} \mathrm{D}=0.19, P<2.2 \times 10^{-16}\right]$.

In agreement with FMRP having a concurrent role in FUSmediated toxicity, FMRP coexpression can rescue FUS-dependent denervation in zebrafish (15). However, because FMRP LLPS behavior, rather than expression levels, is affected by mutant FUS expression, and given that overexpression of FMRP itself promotes its LLPS, we believe that it is unlikely that FMRP overexpression could rescue the translation deficit in our model, although it may have some localized effect on specific targets (52).

Cytoplasmic mislocalization of FUS also occurs in the absence of disease-associated mutations, both in ALS cases caused by other genetic determinants (53) and in cases of frontotemporal lobar degeneration $(54,55)$. In our in vitro translation assays, we show that wild-type FUS impairs translation in a similar manner to ALS mutants (Fig. 3A). Although FUS-ALS mutations may also affect the biophysical properties of the condensates, such as preventing their dissolution (7), and therefore cause a worsening of the phenotype, FUS mislocalization may be sufficient to drive translation repression in these pathologies. Moreover, in our in vitro experiments, FUS ${ }^{\mathrm{WT}}$ and FUS ${ }^{\mathrm{P} 525 \mathrm{~L}}$ have a higher propensity to undergo LLPS compared to $\mathrm{FUS}^{\Delta 14}$ (Fig. 2B), which is likely due to the lack of the C-terminal RGG/RG region (fig. S4C). Because both ALS-mutants cause a very aggressive disease in patients and NLS-lacking FUS mutants, such as FUS $^{\Delta 14}$, have a stronger mislocalization compared to NLS missense mutants, such as FUS ${ }^{\mathrm{P} 525 \mathrm{~L}}$, this highlights how a combination of mislocalization and LLPS behavior can determine FUS cytoplasmic aberrant function and result in comparable cellular toxicity.
In addition to the aforementioned mechanisms, mislocalization of FUS can result in alterations of the protein cellular concentration by affecting its autoregulation and therefore leading to the worsening of its aberrant cytoplasmic functions. Previous studies have shown that FUS-ALS mutations result in increased Fus RNA levels $(51,56)$; we here find Fus to be increased also in the mutant translatome dataset. This further supports that the altered transcript expression indeed results in increased FUS protein translation, which, in turn, could be crucial in increasing its cytoplasmic partitioning and create a vicious cycle leading to increased FUS and FMRP condensate formation.

Our results support a model whereby the presence of cytoplasmic mutant FUS-positive condensates alters the partitioning of a wider network of RBPs, which may affect, in multiple ways, protein translation. While under physiological conditions FMRP is in an equilibrium between a diffuse and a condensed state, increased cytoplasmic levels of FUS due to ALS mutations promote the phase separation of both proteins (Fig. 5, top). We find that wild-type FUS has a critical role in FMRP association with the translational machinery, but because this effect is comparable in both mutant FUS expression and in loss of FUS, it cannot be accounted for the translation repression detected only in mutant FUS-expressing MNs. Instead, our data support a model where increased FMRP condensate formation, induced by mutant FUS, reduces the availability of FMRP-bound RNAs, ultimately altering their translation (Fig. 5). It was recently shown that an altered cross-regulation between FUS, FMRP, and the RBP HuD results in an aberrant axonal phenotype in FUS-ALS models (52). It is therefore likely that FUS and FMRP and possibly other LCR-containing RBPs with a similar biophysical behavior form a wider network and alterations in their cytoplasmic localization can influence their LLPS behavior. This would result in the generation of heterogeneous condensates in which the RNAs that are bound by RBPs present in the condensates are sequestered and their translation inhibited, with a major impact on overall neuronal functionality. FUS mislocalization and the aberrant phase separation of the RBP network can therefore alter neuronal function via a misregulation of finely tuned translational requirements, possibly contributing to ALS pathogenesis and ultimately affecting MN survival.

\section{MATERIALS AND METHODS}

\section{Animals}

$\Delta 14$ Fus mice (B6N;B6)-Fus ${ }^{\text {tm1Emc/H }}$, MGI (Mouse Genome Informatics) MGI:6100933) were previously described (13). Fus knockout mice were obtained from the Mouse Knockout Project [Fustm1(KOMP)Vlcg]. HB9::GFP (green fluorescent protein) [B6.Cg-Tg(Hlxb9-GFP)1Tmj/J], choline acetyltransferase (ChAT)-internal ribosomal entry site (IRES)-Cre [B6;129S6-Chattm2(cre)Lowl/J], and RiboTag (B6N.129Rpl22tm1.1Psam/J) mice were obtained from the Jackson laboratory. All mouse lines were backcrossed onto C57BL/6 J animals for more than five generations. Both $\Delta 14$ Fus knockin and Fus knockout animals were maintained in heterozygosity, because homozygous mice die perinatally. Both mouse lines were crossed with heterozygous HB9::GFP mice when required.

For RiboTag experiments, both RiboTag and ChAT-Cre homozygous mice were crossed with either Fus ${ }^{\mathrm{\Delta 14/+}}$ or $\mathrm{Fus}^{+/-}$animals. Double transgenic mice were subsequently crossed to obtain experimental progeny. 
All experiments were carried out following the guidelines of the UCL (University College London) Queen Square Institute of Neurology Genetic Manipulation and Ethics Committees and in accordance with the European Community Council Directive of 24 November 1986 (86/609/EEC). All procedures for the care and treatment of animals were carried out under license from the U.K. Home Office in accordance with the Animals (Scientific Procedures) Act 1986 Amendment Regulations 2012 and were approved by the UCL Queen Square Institute of Neurology Ethical Review Committee.

\section{Primary MN preparation}

E12.5-14.5 embryos for ventral horn cultures were obtained from heterozygous $\mathrm{Fus}^{\Delta 14 /+}$ or $\mathrm{Fus}^{-1+}$ mice (with or without the HB9::GFP transgene). Briefly, embryos were euthanized, a sample of the tail was used for genotyping, and the body was maintained in ice-cold Hibernate-E media supplemented with B27. Spinal cords of the correct genotype were then dissected, meninges were removed, and dorsal horns were resected. Spinal cord ventral horns were incubated in $0.025 \%$ trypsin for $10 \mathrm{~min}$ at $37^{\circ} \mathrm{C}$. Trypsin was then removed, and the tissue was triturated in L15 media containing $0.4 \%$ bovine serum albumin (BSA) and deoxyribonuclease (DNase, $0.1 \mathrm{mg} / \mathrm{ml}$ ). Neurons were pelleted through a $4 \%$ BSA cushion; resuspended in Neurobasal media (Thermo Fisher Scientific) containing $2 \%$ heatinactivated horse serum, $1 \times$ B27, $1 \times$ GlutaMAX, $1 \times$ penicillin/ streptomycin, $24.8 \mu \mathrm{M} \beta$-mercaptoethanol, brain-derived neurotrophic factor (BDNF, $1 \mathrm{ng} / \mathrm{ml}$ ), glial cell line-derived neurotrophic factor (GDNF, $0.1 \mathrm{ng} / \mathrm{ml}$ ), and ciliary neurotrophic factor (CNTF, $10 \mathrm{ng} / \mathrm{ml}$ ); and immediately plated onto $13-\mathrm{mm}$ coverslips, microfluidic chambers (MFCs), or 3-cm dishes that had been precoated first with poly-ornithine $(10 \mu \mathrm{g} / \mathrm{ml})$ and laminin $(3 \mu \mathrm{g} / \mathrm{ml})$. Neurons were maintained in culture in a humidified incubator at $37^{\circ} \mathrm{C}$ with $5 \% \mathrm{CO}_{2}$ for 5 to 7 DIV. MNs were transfected at DIV 2 by magnetofection as previously described (57).

\section{iPSC maintenance and differentiation}

Human iPSCs used in this study are the isogenic FUS ${ }^{\mathrm{WT} / \mathrm{WT}}$ and $\mathrm{FUS}^{\mathrm{P} 525 \mathrm{~L} / \mathrm{P525L}}$ lines that were derived and maintained as described (58) and differentiated into spinal MNs as described $(35,59)$. Briefly, iPSCs stably transduced with a piggyBac vector carrying inducible Ngn2, Isl1, and Lhx3 (NIL) transgenes dissociated to single cells with Accutase (Thermo Fisher Scientific) and plated in Nutristem $\mathrm{XF} / \mathrm{FF}$ medium (Biological Industries) supplemented with $10 \mu \mathrm{M}$ ROCK inhibitor (Enzo Life Sciences) on Matrigel (BD Biosciences) at a density of $100,000 \mathrm{cells} / \mathrm{cm}^{2}$. The day after NIL expression was induced by adding doxycycline (dox) $(1 \mu \mathrm{g} / \mathrm{ml}$; Thermo Fisher Scientific) in Dulbecco's modified Eagle's medium (DMEM)/F12 medium [DMEM/F12 (Sigma-Aldrich), supplemented with $1 \times$ GlutaMAX (Thermo Fisher Scientific), $1 \times$ nonesssential amino acid (NEAA) (Thermo Fisher Scientific), and $0.5 \times$ penicillin/streptomycin (Sigma-Aldrich)]. The medium was changed every day. After 48 hours of dox induction, the medium was changed to Neurobasal/B27 medium [Neurobasal Medium (Thermo Fisher Scientific), supplemented with $1 \times$ B27 (Thermo Fisher Scientific), $1 \times$ GlutaMAX (Thermo Fisher Scientific), $1 \times$ NEAA (Thermo Fisher Scientific), and $0.5 \times$ penicillin/streptomycin (Sigma-Aldrich)], containing $5 \mu \mathrm{M}$ DAPT and $4 \mu \mathrm{M}$ SU5402 (both from Sigma-Aldrich). At day 5, cells were dissociated with Accutase (Thermo Fisher Scientific) and plated on Matrigel (BD Biosciences)-coated dishes or coverslips at the density of $100,000 \mathrm{cells} / \mathrm{cm}^{2}$. ROCK inhibitor $(10 \mu \mathrm{M})$ was added for the first 24 hours after dissociation. Neuronal cultures were maintained in neuronal medium [Neurobasal/B27 medium supplemented with BDNF $(20 \mathrm{ng} / \mathrm{ml})$ and GDNF $(10 \mathrm{ng} / \mathrm{ml})$ (both from PreproTech) and L-ascorbic acid (20 ng/ml; Sigma-Aldrich)].

\section{MEFs and cell lines}

MEFs were isolated from the embryonic tissue discarded from the primary MN preparation. Viscera were removed, and the remaining tissue was triturated with a blade and incubated in $0.25 \%$ trypsin for $20 \mathrm{~min}$ at $37^{\circ} \mathrm{C}$. Trypsin was quenched, and cells were plated in DMEM (Thermo Fisher Scientific) containing 10\% fetal bovine serum (FBS) and $1 \times$ penicillin/streptomycin. After 3 days in culture, cells were immortalized by transfection with Lipofectamine 3000 (Invitrogen) with the simian virus 40 (SV40) T antigen. After 5 to 7 passages at low density, the cultures presented a homogeneous cell population and started to grow steadily.

HeLa and MEF cells were maintained in DMEM containing $10 \% \mathrm{FBS}$ and $1 \times$ penicillin/streptomycin and were maintained in a humidified incubator at $37^{\circ} \mathrm{C}$ with $5 \% \mathrm{CO}_{2}$. Transfection was performed with Lipofectamine 3000 (Invitrogen) according to the manufacturer's instructions, 0.3 to $0.6 \mu \mathrm{g}$ of DNA per coverslip was used, and cells were analyzed 18 to 48 hours after transfection.

\section{Microfluidic chambers}

MFCs were made with a Sylgard 184 silicone elastomer kit (Dow Corning) using epoxy resin molds previously designed in the laboratory (60). Once the MFCs were baked, reservoirs were cut, and the MFCs were mounted onto glass-bottom dishes (HBST-5040, WillCo Wells), precoated with poly-D-lysine $(20 \mu \mathrm{g} / \mathrm{ml})$. MFCs were then blocked with $0.8 \%$ BSA (BioXtra, Sigma-Aldrich) overnight, polyornithine ( $>3$ hours), and last, laminin (overnight), before plating MNs. MFCs have 500- $\mu \mathrm{m}$-long grooves that separate the somatic from the axonal compartment.

\section{Constructs}

mCherry-FUS ${ }^{513 x}(\Delta N L S)$ was gifted by D. Dormann, and pcDNA6Flag-FUS was gifted by M.-D. Ruepp. pcDNA6-Flag-FUS ${ }^{\mathrm{P} 525 \mathrm{~L}}$ was generated in the laboratory by PCR mutagenesis. pBABE-puro SV40 Large T antigen was a gift from T. Roberts (Addgene plasmid no. 13970; http://n2t.net/addgene:13970; RRID:Addgene_13970).

\section{Antibodies}

The following antibodies were used: anti-FUS N-terminal [western blot (WB) 1:5000; catalog no. NB100-565, Novus Biologicals], anti-FUS C-terminal [immunofluorescence (IF) 1:300, WB 1:5000; catalog no. NB100-562, Novus Biologicals], anti-FUS (IF 1:400; catalog no. sc-47711, Santa Cruz Biotechnology), anti- $\Delta 14$ [IF 1:300 (13)], $\beta 3$-tubulin [1:1000; catalog no. 801202 (BioLegend); 1:500; catalog no. 302306 (SySy); and 1:2000; catalog no. 119-154886 (Raybiotech)] anti-GFP (IF 1:1000; catalog no. GFP1011, Aves Labs), anti-FMRP (IF 1:300 and WB 1:1000; catalog no. ab17722, Abcam), anti-SMN1 (IF 1:300 and WB 1:1000; catalog no. 610646, BD Biosciences), anti-Flag M1 (IF 1:500; catalog no. F3040, Sigma), anti-G3BP1 (1:200; catalog no. 611126, BD Biosciences), anti-RPL26 (IF 1:800 and WB 1:2000; catalog no. ab59567, Abcam), anti-RPS6 (WB 1:1000; Cell Signaling Technology), anti-LAMP1 (IF 1:300; catalog no. ab25245, Abcam), anti-glyceraldehyde-3-phosphate dehydrogenase (GAPDH) (WB 1:5000; mab374, Millipore), anti-HA [WB 1:3000 and immunohistochemistry (IHC) 1:100; catalog no. H6908, Sigma-Aldrich], 
and anti-ChAT (IHC 1:100; Chemicon). Alexa Fluor-conjugated secondary antibodies were from Invitrogen (1:1000) or Jackson ImmunoResearch (1:500).

\section{Immunofluorescence}

Cells were fixed in paraformaldehyde (PFA) solution [4\% PFA and $4 \%$ sucrose in phosphate-buffered saline (PBS)] for $15 \mathrm{~min}$ at room temperature (RT). Samples were then permeabilized and blocked in a solution containing 10\% HRS (Horse serum), 0.5\% BSA, and $0.2 \%$ Triton X-100 in PBS for 15 min. Primary antibodies were diluted in blocking solution (10\% HRS and $0.5 \%$ BSA in PBS) and incubated for 1 hour at RT. Secondary antibodies were diluted in blocking solution (10\% HRS and $0.5 \%$ BSA in PBS) and incubated for 1 hour at RT.

Coverslips were mounted using Mowiol or Fluoromount-G (Thermo Fisher Scientific); MFCs were mounted with Ibidi mounting media. Imaging was carried out using a Zeiss LSM 780 inverted confocal microscope with a $40 \times$ oil immersion lens with 1.3 numerical aperture or with a Zeiss LSM 710 inverted confocal microscope with a $63 \times$ oil immersion lens with 1.4 numerical aperture. Images were digitally captured using ZEN 2010 software and analyzed using Fiji (ImageJ).

\section{Immunohistochemistry}

For immunohistochemical analysis, 3-month-old mice were perfused with saline, followed by $4 \%$ PFA solution. Spinal cords were dissected, incubated in $20 \%$ (w/v) sucrose, embedded in Tissue-Tek optimum cutting temperature compound (OCT) (Sakura Finetek, 4583), and sectioned with an OTF cryostat (Bright Instruments). Slices were mounted on microscope slides, and sections were encircled with a hydrophobic barrier pen (Dako, S2002), permeabilized by three 10 -min washes with $0.3 \%$ Triton X-100 in PBS, and blocked for 1 hour in $10 \%$ BSA and $0.3 \%$ Triton X-100 in PBS. Samples were then probed with primary antibodies overnight; the samples were then washed three times before incubation with secondary antibodies for 1 hour. Slides were then washed, mounting media were added, and samples were covered with $22 \mathrm{~mm}$ by $50 \mathrm{~mm}$ cover glass.

\section{Cellular translation assay}

AHA labeling assays were carried out as previously described (61). Briefly, neurons were incubated in a neuronal methionine-free media consisting of methionine and cysteine-free DMEM supplemented with $0.26 \mathrm{mM}$ L-cysteine, $0.23 \mathrm{mM}$ sodium pyruvate, $10 \mathrm{mM}$ HEPES ( $\mathrm{pH}$ 7.4), $0.067 \mathrm{mM}$ L-proline, $0.674 \mu \mathrm{M}$ zinc sulfate, $5 \mathrm{nM} \mathrm{B12,} 1 \times$ GlutaMAX, $1 \times$ B27, $1 \times$ penicillin/streptomycin, BDNF $(1 \mathrm{ng} / \mathrm{ml})$, GDNF $(0.1 \mathrm{ng} / \mathrm{ml})$, and CNTF $(10 \mathrm{ng} / \mathrm{ml})$ for $30 \mathrm{~min}$ before the addition of $2 \mathrm{mM}$ AHA or vehicle control for $30 \mathrm{~min}$. Anisomycin $(40 \mu \mathrm{M})$ was preincubated for $20 \mathrm{~min}$ and coincubated with AHA. Neurons were then fixed and permeabilized, and AHA was labeled by click chemistry using the Click-iT Cell Reaction Buffer Kit with an Alexa Fluor 555 alkyne $(1 \mu \mathrm{M})$ following the manufacturer's instructions.

\section{Proximity ligation assays}

PLA was performed with Duolink In Situ Orange PLA reagents according to the manufacturer's protocol (Sigma-Aldrich).

\section{Image analysis}

Images were analyzed using Fiji (ImageJ). Digital deconvolution was performed using the following plug-ins: "Diffraction PSF 3D" was used to generate a theoretical point spread function for each wavelength, and "Parallel spectral deconvolution 2D" was used for the generation of the deconvolved image. Puncta number was quantified manually using the "Cell Counter" plug-in. SynPAnal (62) was used to quantify axonal puncta size. PLAs were analyzed by using Fiji particle analysis and PLA puncta with sizes comprising 0.05 to $3.00 \mu \mathrm{m}$ were quantified. In translation assays, MNs were selected by HB9::GFP expression and imaged. The AHA-Alexa Fluor 555 signal within $\mathrm{MN}$ cell bodies was quantified as mean fluorescence intensity. All analyses were performed on blinded samples.

\section{Live imaging and analysis}

Live axonal transport assays were performed in motor neuronal cultures grown in MFCs at DIV 7 to 8 as previously described (63). Briefly, for signaling endosomes and mitochondria transport assays, neurons were incubated with either $30 \mathrm{nM}$ Alexa Fluor 555-HcT or $125 \mathrm{nM}$ MitoTracker Deep Red (Molecular Probes, Invitrogen) for $30 \mathrm{~min}$ at $37^{\circ} \mathrm{C}$. Cells were then washed, and new MN media containing $20 \mathrm{mM}$ HEPES-NaOH (pH 7.4) were added. After 15 min, transport was assessed at $37^{\circ} \mathrm{C}$ using an inverted Zeiss LSM 780 microscope equipped with a Zeiss $40 \times, 1.3$ numerical aperture differential interference contrast Plan-Apochromat oil immersion objective. Images were taken at $2 \mathrm{~Hz}$ for 2 to $4 \mathrm{~min}$. Cargoes were tracked using the Fiji plug-in TrackMate (64), and data analysis was performed in R.

\section{Western blotting and coimmunoprecipitation}

$\mathrm{MN}$ cultures were lysed in radioimmunoprecipitation assay buffer (RIPA) [50 mM tris- $\mathrm{HCl}$ (pH 7.5), $150 \mathrm{mM} \mathrm{NaCl,} \mathrm{1 \%} \mathrm{NP-40,} \mathrm{0.5 \%}$ sodium deoxycholate, $0.1 \%$ SDS, 1 mM EDTA, 1 mM EGTA, Halt phosphatase, and protease inhibitor cocktail (Thermo Fisher Scientific)], incubated on a rotating wheel at $4^{\circ} \mathrm{C}$ for 1 hour, and then nuclei and cellular debris were spun down at $20,000 \mathrm{~g}$ for $10 \mathrm{~min}$. Supernatants were collected, Laemmli buffer was added, and samples were denatured at $98^{\circ} \mathrm{C}$ for $5 \mathrm{~min}$.

For coimmunoprecipitation (co-IP) assays, E12.5-14.5 brains were used. Samples were homogenized in lysis buffer [20 mM HEPES (pH 7.4), $150 \mathrm{mM} \mathrm{NaCl}, 10 \%$ glycerol, Halt phosphatase, and protease inhibitor cocktail] and incubated on a rotating wheel at $4^{\circ} \mathrm{C}$ for 1 hour; nuclei and cellular debris were spun down at $20,000 \mathrm{~g}$ for $20 \mathrm{~min}$. Supernatants were collected, $1 \mathrm{mg}$ of protein lysate was used per co-IP, and protein of interest was immunoprecipitated with $2 \mu \mathrm{g}$ of antibody or appropriate immunoglobulin G control overnight. Protein A Sepharose beads (Sigma-Aldrich) were used to purify the antibody/protein complex; precipitates were washed three times before being eluted in Laemmli buffer.

Samples were separated on precast 4 to $15 \%$ Mini-PROTEAN TGX Stain-Free protein gels (Bio-Rad) and transferred onto a polyvinylidene difluoride membrane using a semidry Trans-Blot Turbo system (Bio-Rad), or NuPAGE 4 to $12 \%$ bis-tris protein gels were used, and proteins were blotted onto a nitrocellulose membrane using a Novex system (GE Healthcare). Western blots were developed with a Classico substrate (Millipore) and detected with a ChemiDoc imaging system (Bio-Rad). Densitometric quantification of bands was carried out using the software Image Lab (Bio-Rad).

\section{Polysome profiling}

Cytoplasmic lysates from frozen E17.5 brains were prepared as described previously (29). Tissue was pulverized in a mortar under liquid nitrogen. The tissue powder was dissolved in $10 \mathrm{mM}$ tris- $\mathrm{HCl}$ ( $\mathrm{pH}$ 7.5), $10 \mathrm{mM} \mathrm{NaCl}, 10 \mathrm{mM} \mathrm{MgCl}, 1 \%$ Triton X-100, $1 \%$ sodium 
deoxycholate, RiboLock RNase Inhibitor $(0.4 \mathrm{U} / \mathrm{ml}$; Thermo Fisher Scientific), $1 \mathrm{mM}$ dithiothreitol, cycloheximide $(0.2 \mathrm{mg} / \mathrm{ml})$, and DNase I ( $5 \mathrm{U} / \mathrm{ml}$; Thermo Fisher Scientific). Following a first centrifugation step for $1 \mathrm{~min}$ at $14,000 \mathrm{~g}$ at $4^{\circ} \mathrm{C}$ to remove tissue debris, the supernatant was centrifuged for $5 \mathrm{~min}$ at $14,000 \mathrm{~g}$ to pellet nuclei and mitochondria. Cleared supernatants were then loaded on a linear 15 to $50 \%$ sucrose gradient in $10 \mathrm{mM}$ tris- $\mathrm{HCl}$ ( $\mathrm{pH} 7.5), 100 \mathrm{mM}$ $\mathrm{NaCl}$, and $10 \mathrm{mM} \mathrm{MgCl}_{2}$ and ultracentrifuged in a SW41Ti rotor (Beckman) for 1 hour and $40 \mathrm{~min}$ at $40,000 \mathrm{rpm}$ at $4^{\circ} \mathrm{C}$ in a Beckman Optima LE-80 K ultracentrifuge. After ultracentrifugation, gradients were fractionated in $1-\mathrm{ml}$ volume fractions with continuous monitoring absorbance at $254 \mathrm{~nm}$ using an ISCO UA-6 ultraviolet detector. Proteins were extracted from each sucrose fraction of the profile using the methanol/chloroform protocol and solubilized in a sample buffer.

\section{Cloning and purification of MBP-FUS and mutants}

MBP-FUS was a gift from N. Fawzi (Addgene, plasmid no. 98651; http://n2t.net/addgene:98651; RRID:Addgene_98651). The P525L FUS point mutation and the $\Delta 14$ mutant version of FUS were generated via site-directed mutagenesis using MBP-FUS. The amino acid sequence at the $\mathrm{C}$ terminus of the $\Delta 14$ mutant is "KAPKPDGPGGGPGGSHMGVSTDRIAGRGRIN*”.

MBP-FUS and mutants were expressed in Escherichia coli BL21 DE3 cells with rare codons for R, I, P, and L using chloramphenicol and kanamycin for selection. Following cell lysis by sonication, the protein was purified by nickel-affinity chromatography. The lysis buffer used contained $20 \mathrm{mM}$ sodium phosphate, $500 \mathrm{mM} \mathrm{NaCl}$, $5 \mathrm{mM} \beta$-mercaptoethanol, and $20 \mathrm{mM}$ imidazole ( $\mathrm{pH} \mathrm{7.4).} \mathrm{One}$ Complete Protease Inhibitor tablet (Sigma-Aldrich) was added to the lysate from 2 liters of growth. The column was washed with the same buffer supplemented with $40 \mathrm{mM}$ imidazole. Protein was eluted in lysis buffer with $400 \mathrm{mM}$ imidazole. The protein was then further purified using gel filtration chromatography with a buffer containing $50 \mathrm{mM}$ tris- $\mathrm{HCl}$ (pH 7.6), $150 \mathrm{mM} \mathrm{NaCl}, 5 \mathrm{mM} \beta$-mercaptoethanol, and $1 \mathrm{mM}$ EDTA.

\section{Protein expression and purification of FMRP-C-terminal}

The low-complexity disordered region of human FMRP $445-632$ (referred to as $\mathrm{FMRP}_{\mathrm{LCR}}$ ) was expressed and purified as previously described (23). Briefly, His-Small Ubiquitin-like Modifier (SUMO)FMRP was transformed into E. coli BL21-CodonPlus(DE3) RIL cells and grown at $37^{\circ} \mathrm{C}$ in LB. Protein expression was induced with $0.5 \mathrm{mM}$ isopropyl- $\beta$-D-thiogalactopyranoside (IPTG) at an $\mathrm{OD}_{600}$ (optical density at $600 \mathrm{~nm}) \sim 0.6$ and grown overnight at $25^{\circ} \mathrm{C}$. Cells were harvested and lysed in lysis buffer containing $6 \mathrm{M}$ guanidinium chloride (GdnHCl), $50 \mathrm{mM}$ tris- $\mathrm{HCl}$ ( $\mathrm{pH}$ 8.0), $500 \mathrm{mM} \mathrm{NaCl}, 20 \mathrm{mM}$ imidazole, and $2 \mathrm{mM} \beta$-mercaptoethanol. Harvested cells were sonicated for $4.5 \mathrm{~min}$ ( $2 \mathrm{~s}$ on, $1 \mathrm{~s}$ off) and centrifuged. The supernatant of the lysate was then purified by nickel-affinity chromatography equilibrated with the lysis buffer. The column was washed in lysis buffer without $6 \mathrm{M} \mathrm{GdnHCl}$ and then eluted in buffer containing $50 \mathrm{mM}$ tris- $\mathrm{HCl}$ (pH 8.0), $500 \mathrm{mM} \mathrm{NaCl}, 300 \mathrm{mM}$ imidazole, and $2 \mathrm{mM}$ $\beta$-mercaptoethanol. The His-SUMO tag was cleaved with ubiquitinlike protease, while dialyzed against cleavage buffer $(50 \mathrm{mM}$ tris- $\mathrm{HCl}$, $150 \mathrm{mM} \mathrm{NaCl}, 20 \mathrm{mM}$ imidazole, and $2 \mathrm{mM} \beta$-mercaptoethanol at $\mathrm{pH}$ 7.4) overnight at $4^{\circ} \mathrm{C}$. FMRP was separated from the His-SUMO tag by nickel-affinity chromatography following the same steps described above. The fractions containing FMRP were collected, and successful separation of FMRP from the His-SUMO tag was verified with SDS-polyacrylamide gel electrophoresis (SDS-PAGE) gel. FMRP was concentrated and further purified using gel filtration chromatography with a buffer containing $4 \mathrm{M} \mathrm{GdnHCl,} 50 \mathrm{mM}$ tris- $\mathrm{HCl}$ (pH 8.0), $500 \mathrm{mM} \mathrm{NaCl}$, and $2 \mathrm{mM} \beta$-mercaptoethanol.

\section{Fluorescence protein labeling}

An Alexa Fluor 647 fluorescent dye was added to the only cysteine (C584) in FMRP $445-632$ via a maleimide linkage following the manufacturer's instruction with slight modifications. First, FMRP was dialyzed into a buffer containing $50 \mathrm{mM}$ tris- $\mathrm{HCl}$ (pH 7.5), $100 \mathrm{mM}$ $\mathrm{NaCl}$, and $4 \mathrm{M} \mathrm{GdnHCl}$. To ensure that any residual reducing agents were removed, the protein was desalted using a Hi-Trap desalting column (GE Healthcare). After desalting, the protein sample was immediately reacted with $5 \times$ Alexa Fluor 647 (Thermo Fisher Scientific) maleimide dye. The reaction was incubated overnight at $4^{\circ} \mathrm{C}$ and quenched with an excess of reducing agent [dithiothreitol (DTT)] the following day. To remove any unreacted dye, the protein was passed through a Hi-Trap desalting column (GE Healthcare) and an S75 gel filtration column equilibrated in buffer containing $50 \mathrm{mM}$ tris- $\mathrm{HCl}$ (pH 7.5), $100 \mathrm{mM} \mathrm{NaCl}, 4 \mathrm{M} \mathrm{GdnHCl}$, and $2 \mathrm{mM}$ DTT. Successful dye separation was confirmed by running the protein sample on an SDS-PAGE gel and then visualizing any remaining free dye with a fluorescence reader, ChemiDoc MP System (Bio-Rad). Labeled proteins were either frozen or dialyzed into specific assay buffers.

\section{RNA preparation}

Sc1 RNA (GCUGCGGUGUGGAAGGAGUGGUCGGGUUGCGCAGCG) was purchased from Sigma-Aldrich as lyophilized samples. Stocks $(100 \mu \mathrm{M})$ were reconstituted in water and stored at $-20^{\circ} \mathrm{C}$. Working stocks were diluted into specific assay buffers.

\section{Turbidity measurements}

For turbidity measurements, $\mathrm{OD}_{600}$ of MBP-FUS was obtained using a SpectraMax i3× multimode plate reader (Molecular Devices) at $25^{\circ} \mathrm{C}$. The samples were prepared by mixing varying concentrations of MBP-FUS with $0.5 \mu \mathrm{M}$ TEV protease in a buffer containing $25 \mathrm{mM}$ tris- $\mathrm{HCl}$ ( $\mathrm{pH} 7.4$ ), $150 \mathrm{mM} \mathrm{KCl}$, and $2 \mathrm{mM}$ DTT. Samples were equilibrated for 5 min before reading the turbidity. Turbidity was measured at intervals of $35 \mathrm{~s}$ for a total of $20 \mathrm{~min}$. The change in turbidity was calculated from the slope ( $\Delta$ absorbance/min) from 0 to $5 \mathrm{~min}$. Apparent $C_{\text {sats }}$ are calculated as previously described (48).

\section{In vitro co-LLPS assays}

In vitro phase separation assays of LCRs of FUS and FMRP were performed using ${ }^{\text {FITC }_{\text {FUS }}}$ LCR $(50 \mu \mathrm{M})$ and ${ }^{\text {Alexa647 }}{ }^{\text {FMRP }}$ LCR $_{\text {LC }}(50 \mu \mathrm{M})$ in the presence of $1 \mu \mathrm{M} \mathrm{scl}$ RNA in a buffer containing $25 \mathrm{mM}$ sodium phosphate ( $\mathrm{pH} 7.4$ ), $50 \mathrm{mM} \mathrm{KCl}$, and $2 \mathrm{mM}$ DTT. For partitioning assays using full-length FUS proteins (FUS ${ }^{\mathrm{WT}}$, FUS ${ }^{\mathrm{P} 525 \mathrm{~L}}$, and FUS $\left.{ }^{\Delta 14}\right)$, MBP-FUS $(10 \mu \mathrm{M})$ phase separation was induced by TEV protease $(0.5 \mu \mathrm{M})$ cleavage before the addition of ${ }^{\text {Alexa647 }} \mathrm{FMRP}_{\mathrm{LCR}}$ $(1 \mu \mathrm{M})$ and sc1 RNA $(0.5 \mu \mathrm{M})$ in a buffer containing $25 \mathrm{mM}$ sodium phosphate (pH 7.4), $150 \mathrm{mM} \mathrm{KCl}$, and 2 mM DTT.

\section{Fluorescence microscopy of phase-separated samples}

Fluorescence images of phase-separated droplets were imaged on a confocal Leica DMi8 microscope equipped with a Hamamatsu 
C9100-13 electron multiplying charge-coupled device (EM-CCD) camera with a $63 \times$ objective. Alexa Fluor 647 fluorescence was detected using a 637-nm laser, and FITC fluorescence was detected using a 491-nm laser. In experiments with MBP-FUS and MBP-FUS mutants, samples were incubated with TEV protease for $10 \mathrm{~min}$ before imaging. All phase-separated droplets were imaged on a 96-well glass plate (Eppendorf). Two- or threefold concentrated protein or RNA samples were prepared to account for the dilution in mixing with other components to achieve desired final concentrations. Note that no molecular crowding reagents were used. Images represent droplets settled to the bottom of the plate. The images were processed using Volocity (PerkinElmer) and ImageJ.

\section{In vitro partitioning assay}

To determine the partitioning of FMRP, images of droplets with the addition of $5 \%{ }^{\text {Alexa647 }}{ }^{\text {FMRP }}$ LCR were acquired as described above and analyzed with Image J. An image of the buffer in the absence of any protein was used to subtract any background artifacts. In ImageJ, masks were defined using the Otsu threshold method while applying several criteria to the particle picking algorithm: droplets are required to have a radius greater than $1 \mu \mathrm{m}$ and with the circularity of 0.5 to 1.0. The intensity of the bulk background solution is defined as the mean intensity within a circular region of interest with a diameter of $5 \mu \mathrm{m}$ that does not contain any phase-separated droplets. Fluorescence enrichment ratios were calculated from the ratio of the mean fluorescence intensity (inside droplet)/mean fluorescence intensity (background outside of droplet). Droplets were randomly imaged, and measurement represents three independent experiments.

\section{In vitro translation assay}

In vitro translation rates represent the increase in luminescence as a function of time using a standard rabbit reticulocyte lysate system (Promega) with luciferase mRNA (Promega). Manufacturer's instructions were followed with a few modifications. Briefly, each reaction $(30 \mu \mathrm{l})$ contains $12.6 \mu \mathrm{l}$ of rabbit reticulocyte lysate, $0.5 \mu \mathrm{l}$ of luciferase mRNA ( $1 \mathrm{mg} / \mathrm{ml}), 0.3 \mu \mathrm{l}$ of amino acid mixture minus leucine $(1 \mathrm{mM}), 0.3 \mu \mathrm{l}$ of amino acid mixture minus methionine $(1 \mathrm{mM}), 2 \mu \mathrm{l}$ of TEV protease $(15 \mu \mathrm{M})$, and $14.3 \mu \mathrm{l}$ of $5 \mu \mathrm{M}$ protein (MBP-FUS/FMRP) or buffer [25 mM sodium phosphate ( $\mathrm{pH} 7.4$ ), $50 \mathrm{mM} \mathrm{KCl}$, and $2 \mathrm{mM}$ DTT). First, the reaction was incubated for $10 \mathrm{~min}$, and then, end-point luminescence measurements were carried out in intervals of $10 \mathrm{~min}$ up to $50 \mathrm{~min}$. Each end-point luminescence measurement contained $75 \mu \mathrm{l}$ of luciferase substrate mixed with $2.5 \mu \mathrm{l}$ of unpurified translation mixture measured in a white opaque 96-well plate (Corning 3990). A SpectraMax i3× multimode plate reader (Molecular Devices) at $25^{\circ} \mathrm{C}$ was used to detect the luminescence. The translation rates represent the line of best fit from the end-point luminescence readings as a function of time.

\section{RiboTag}

The RiboTag method was performed as described previously (65) with modifications. Briefly, E17.5 spinal cords were homogenized using TissueRuptor (Qiagen) in a buffer [ $50 \mathrm{mM}$ tris- $\mathrm{HCl}$ ( $\mathrm{pH} 7.4$ ), $100 \mathrm{mM} \mathrm{KCl}, 12 \mathrm{mM} \mathrm{MgCl}$, and $1 \% \mathrm{NP}-40$ ] supplemented with cycloheximide $(0.1 \mathrm{mg} / \mathrm{ml})$, heparin $(1 \mathrm{mg} / \mathrm{ml})$, SuperaseIn RNase (Thermo Fisher Scientific). Lysates were cleared by centrifugation at $10,000 \mathrm{~g}$ for $10 \mathrm{~min}$, and $5 \%$ of the lysate was saved as input. To reduce nonspecific-binding protein $\mathrm{G}$ magnetic beads (Dynabeads, Thermo Fisher Scientific) were added to the lysate and incubated for 2 hours at $4^{\circ} \mathrm{C}$. Next, $5 \mu \mathrm{l}$ of anti-HA antibody (Sigma-Aldrich) was added to the precleared lysate and incubated for 2 hours at $4^{\circ} \mathrm{C}$. Later, $100 \mu \mathrm{l}$ of beads slurry with $2 \mu \mathrm{l}$ of SuperaseIn were added to the lysate followed by 2-hour incubation in the cold room. After precipitation, beads were washed five times in the wash buffer $[300 \mathrm{mM}$ $\mathrm{KCl}, 1 \% \mathrm{NP}-40,50 \mathrm{mM}$ tris- $\mathrm{HCl}$ (pH 7.4), $12 \mathrm{mM} \mathrm{MgCl}_{2}$, and cycloheximide $(0.1 \mathrm{mg} / \mathrm{ml})]$. Beads were eluted in Qiazol (Qiagen), and RNAs were isolated using the RNeasy Micro Kit (Qiagen). Ten percent of the beads were used for WB and eluted in Laemmli sample buffer (Thermo Fisher Scientific) supplemented with $100 \mathrm{mM}$ DTT. The quality control of RNA was performed using TapeStation (Agilent).

\section{qPCR analysis of RiboTag samples}

SuperScript IV VILO (Thermo Fisher Scientific) was used to reversetranscribe RNA from input and IP samples (concentrations adjusted). Complementary DNA was used for qPCR analysis; expression of Gapdh was used as housekeeping control. Primers used for qPCR analysis are as follows: Chat, GCGTAACAGCCCAGGAGAG (forward) and TTGTACAGGCATCTTTGGGG (reverse); Gapdh, CAAGCTCATTTCCTGGTATGA (forward) and CTCTTGCTCAGTGTCCTTGCT (reverse); HA-Rpl22, GTGCCTTTCTCCAAAAGGTATTT (forward) and GTCATATGGATAGGATCCTGCATA (reverse); and Pmp22, GCCGTCCAACACTGCTACTC (forward) and GAGCTGGCAGAAGAACAGGA (reverse).

\section{RNA sequencing and analysis of RiboTag samples}

Libraries were prepared using NEBNext mRNA Ultra II in the UCL Genomics facility and sequenced (75 base pairs single end) to an average depth of 18 million reads. Each sample was aligned to the Mus musculus (house mouse) genome assembly GRCm38 (mm10) with STAR (v2.4.2a) (66). Reads were coordinate-sorted and marked for PCR duplicates using Novosort (1.03.09). Gene expression was quantified using HTSeq using the Ensembl mm10 (v82) mouse transcript reference (67). Differential gene expression was calculated using DESeq2 (68) comparing the IP samples between the $\mathrm{Fus}^{-/-}$ $(n=4)$ and $F u s^{\Delta 14 / \Delta 14}(n=5)$ with the same number of their respective littermate controls, in two separate analyses. The significance level was set at a false discovery rate-adjusted $P$ value of $10 \%$. To compare the two analyses, each nominal $P$ value was converted into a $z$ score and given the sign of the $\log _{2}$ fold change. We defined condition-specific genes as having an adjusted $P$ value of $<0.1$ in one condition and $>0.1$ in the other. A list of FMRP target genes was obtained from a published HITS (high-throughput sequencing)-CLIP experiment (30). Entrez IDs were converted to Ensembl IDs using $\mathrm{g}$ :Convert from the g:Profiler suite of tools (69). A list of FUS target peaks was obtained from a published iCLIP experiment (32). Peaks were annotated using annotatr (70) using gene models included in the TxDb.Mmusculus.UCSC.mm9.knownGene and org.Mm.eg.db $\mathrm{R}$ packages $(71,72)$. FUS targets were filtered to only use peaks overlapping coding and UTR regions. GO terms analysis was performed in $\mathrm{R}$ using the enrichGO function from the clusterProfiler package (73).

\section{Statistical analysis}

Unless otherwise stated, data were obtained using cells from at least three independent preparations, which are visualized in different shades of gray in the graphs. The number of cells studied is given in the figure legends. GraphPad Prism or R was used for statistical analysis. Normality of data distribution was tested using D'Agostino and 
Pearson normality test. One-way analysis of variance (ANOVA), followed by Dunnet's post hoc test, was used for normally distributed data and multiple comparisons, while Sidak's post hoc test was used for pairwise comparisons. Kruskal-Wallis, followed by Dunn's post hoc test, was used for not normally distributed data and multiple comparisons. Friedman's test, followed by Dunn's post hoc test, was used to compare normally distributed paired samples. Individual differences were assessed using individual Student's $t$ tests. Data are shown as means \pm SEM. Kolmogorov-Smirnov test was used in cumulative frequency analysis to test differences between targets and nontargets of FUS and FMRP.

\section{SUPPLEMENTARY MATERIALS}

Supplementary material for this article is available at http://advances.sciencemag.org/cgi/ content/full/7/30/eabf8660/DC1

View/request a protocol for this paper from Bio-protocol.

\section{REFERENCES AND NOTES}

1. H. Ederle, D. Dormann, TDP-43 and FUS en route from the nucleus to the cytoplasm. FEBS Lett. 591, 1489-1507 (2017).

2. N. Birsa, M. P. Bentham, P. Fratta, Cytoplasmic functions of TDP-43 and FUS and their role in ALS. Semin. Cell Dev. Biol. 99, 193-201 (2020).

3. D. Dormann, R. Rodde, D. Edbauer, E. Bentmann, I. Fischer, A. Hruscha, M. E. Than, I. R. A. Mackenzie, A. Capell, B. Schmid, M. Neumann, C. Haass, ALS-associated fused in sarcoma (FUS) mutations disrupt transportin-mediated nuclear import. EMBO J. 29, 2841-2857 (2010).

4. J. Scekic-Zahirovic, O. Sendscheid, H. El Oussini, M. Jambeau, Y. Sun, S. Mersmann, M. Wagner, S. Dieterlé, J. Sinniger, S. Dirrig-Grosch, K. Drenner, M.-C. Birling, J. Qiu, Y. Zhou, H. Li, X.-D. Fu, C. Rouaux, T. Shelkovnikova, A. Witting, A. C. Ludolph, F. Kiefer, E. Storkebaum, C. Lagier-Tourenne, L. Dupuis, Toxic gain of function from mutant FUS protein is crucial to trigger cell autonomous motor neuron loss. EMBO J. 35, 1077-1097 (2016).

5. C. Vance, B. Rogelj, T. Hortobágyi, K. J. D. Vos, A. L. Nishimura, J. Sreedharan, X. Hu, B. Smith, D. Ruddy, P. Wright, J. Ganesalingam, K. L. Williams, V. Tripathi, S. Al-Saraj, A. Al-Chalabi, P. N. Leigh, I. P. Blair, G. Nicholson, J. de Belleroche, J.-M. Gallo, C. C. Miller, C. E. Shaw, Mutations in FUS, an RNA processing protein, cause familial amyotrophic lateral sclerosis type 6. Science 323, 1208-1211 (2009).

6. L. Guo, H. J. Kim, H. Wang, J. Monaghan, F. Freyermuth, J. C. Sung, K. O’Donovan, C. M. Fare, Z. Diaz, N. Singh, Z. C. Zhang, M. Coughlin, E. A. Sweeny, M. E. DeSantis, M. E. Jackrel, C. B. Rodell, J. A. Burdick, O. D. King, A. D. Gitler, C. Lagier-Tourenne, U. B. Pandey, Y. M. Chook, J. P. Taylor, J. Shorter, Nuclear-import receptors reverse aberrant phase transitions of RNA-binding proteins with prion-like domains. Cell 173 677-692.e20 (2018).

7. T. Murakami, S. Qamar, J. Q. Lin, G. S. K. Schierle, E. Rees, A. Miyashita, A. R. Costa, R. B. Dodd, F. T. S. Chan, C. H. Michel, D. Kronenberg-Versteeg, Y. Li, S.-P. Yang, Y. Wakutani, W. Meadows, R. R. Ferry, L. Dong, G. G. Tartaglia, G. Favrin, W.-L. Lin, D. W. Dickson, M. Zhen, D. Ron, G. Schmitt-Ulms, P. E. Fraser, N. A. Shneider, C. Holt, M. Vendruscolo, C. F. Kaminski, P. S. George-Hyslop, ALS/FTD mutation-induced phase transition of FUS liquid droplets and reversible hydrogels into irreversible hydrogels impairs RNP granule function. Neuron 88, 678-690 (2015).

8. A. G. Niaki, J. Sarkar, X. Cai, K. Rhine, V. Vidaurre, B. Guy, M. Hurst, J. C. Lee, H. R. Koh, L. Guo, C. M. Fare, J. Shorter, S. Myong, Loss of dynamic RNA interaction and aberrant phase separation induced by two distinct types of ALS/FTD-linked FUS mutations. Mol. Cell 77, 82-94.e4 (2020).

9. M. Hofweber, S. Hutten, B. Bourgeois, E. Spreitzer, A. Niedner-Boblenz, M. Schifferer, M.-D. Ruepp, M. Simons, D. Niessing, T. Madl, D. Dormann, Phase separation of FUS is suppressed by its nuclear import receptor and arginine methylation. Cell 173, 706-719. e13 (2018).

10. S. Qamar, G. Wang, S. J. Randle, F. S. Ruggeri, J. A. Varela, J. Q. Lin, E. C. Phillips, A. Miyashita, D. Williams, F. Ströhl, W. Meadows, R. Ferry, V. J. Dardov, G. G. Tartaglia, L. A. Farrer, G. S. Kaminski Schierle, C. F. Kaminski, C. E. Holt, P. E. Fraser, G. Schmitt-Ulms, D. Klenerman, T. Knowles, M. Vendruscolo, P. St George-Hyslop, FUS phase separation is modulated by a molecular chaperone and methylation of arginine cation- $\pi$ interactions. Cell 173, 720-734.e15 (2018).

11. A. Patel, H. O. Lee, L. Jawerth, S. Maharana, M. Jahnel, M. Y. Hein, S. Stoynov, J. Mahamid, S. Saha, T. M. Franzmann, A. Pozniakovski, I. Poser, N. Maghelli, L. A. Royer, M. Weigert, E. W. Myers, S. Grill, D. Drechsel, A. A. Hyman, S. Alberti, A liquid-to-solid phase transition of the ALS protein FUS accelerated by disease mutation. Cell 162, 1066-1077 (2015).
12. A. Bampton, L. M. Gittings, P. Fratta, T. Lashley, A. Gatt, The role of hnRNPs in frontotemporal dementia and amyotrophic lateral sclerosis. Acta Neuropathol. 140, 599-623 (2020).

13. A. Devoy, B. Kalmar, M. Stewart, H. Park, B. Burke, S. J. Noy, Y. Redhead, J. Humphrey, K. Lo, J. Jaeger, A. Mejia Maza, P. Sivakumar, C. Bertolin, G. Soraru, V. Plagnol, L. Greensmith, A. Acevedo Arozena, A. M. Isaacs, B. Davies, P. Fratta, E. M. C. Fisher, Humanized mutant FUS drives progressive motor neuron degeneration without aggregation in "FUSDelta14" knockin mice. Brain 140, 2797-2805 (2017).

14. M. DeJesus-Hernandez, J. Kocerha, N. Finch, R. Crook, M. Baker, P. Desaro, A. Johnston, N. Rutherford, A. Wojtas, K. Kennelly, Z. K. Wszolek, N. Graff-Radford, K. Boylan, R. Rademakers, De novo truncating FUS gene mutation as a cause of sporadic amyotrophic lateral sclerosis. Hum. Mutat. 31, E1377-E1389 (2010).

15. A. M. Blokhuis, M. Koppers, E. J. N. Groen, D. M. A. van den Heuvel, S. Dini Modigliani, J. J. Anink, K. Fumoto, F. van Diggelen, A. Snelting, P. Sodaar, B. M. Verheijen, J. A. A. Demmers, J. H. Veldink, E. Aronica, I. Bozzoni, J. den Hertog, L. H. van den Berg, R. J. Pasterkamp, Comparative interactomics analysis of different ALS-associated proteins identifies converging molecular pathways. Acta Neuropathol. 132, 175-196 (2016).

16. E. J. N. Groen, K. Fumoto, A. M. Blokhuis, J. Engelen-Lee, Y. Zhou, D. M. A. van den Heuvel, M. Koppers, F. van Diggelen, J. van Heest, J. A. A. Demmers, J. Kirby, P. J. Shaw, E. Aronica, W. G. M. Spliet, J. H. Veldink, L. H. van den Berg, R. J. Pasterkamp, ALS-associated mutations in FUS disrupt the axonal distribution and function of SMN. Hum. Mol. Genet. 22, 3690-3704 (2013)

17. S. Sun, S.-C. Ling, J. Qiu, C. P. Albuquerque, Y. Zhou, S. Tokunaga, H. Li, H. Qiu, A. Bui, G. W. Yeo, E. J. Huang, K. Eggan, H. Zhou, X.-D. Fu, C. Lagier-Tourenne, D. W. Cleveland, ALS-causative mutations in FUS/TLS confer gain and loss of function by altered association with SMN and U1-snRNP. Nat. Commun. 6, 6171 (2015).

18. T. Yamazaki, S. Chen, Y. Yu, B. Yan, T. C. Haertlein, M. A. Carrasco, J. C. Tapia, B. Zhai, R. Das, M. Lalancette-Hebert, A. Sharma, S. Chandran, G. Sullivan, A. L. Nishimura, C. E. Shaw, S. P. Gygi, N. A. Shneider, T. Maniatis, R. Reed, FUS-SMN protein interactions link the motor neuron diseases ALS and SMA. Cell Rep. 2, 799-806 (2012).

19. K. Yasuda, H. Zhang, D. Loiselle, T. Haystead, I. G. Macara, S. Mili, The RNA-binding protein Fus directs translation of localized mRNAs in APC-RNP granules. J. Cell Biol. 203, 737-746 (2013).

20. Q. He, W. Ge, The tandem Agenet domain of fragile $X$ mental retardation protein interacts with FUS. Sci. Rep. 7, 962 (2017).

21. J. N. Sleigh, A. P. Tosolini, D. Gordon, A. Devoy, P. Fratta, E. M. C. Fisher, K. Talbot, G. Schiavo, Mice carrying ALS mutant TDP-43, but not mutant FUS, display in vivo defects in axonal transport of signaling endosomes. Cell Rep. 30, 3655-3662.e2 (2020).

22. A. T. Phan, V. Kuryavyi, J. C. Darnell, A. Serganov, A. Majumdar, S. Ilin, T. Raslin, A. Polonskaia, C. Chen, D. Clain, R. B. Darnell, D. J. Patel, Structure-function studies of FMRP RGG peptide recognition of an RNA duplex-quadruplex junction. Nat. Struct. Mol. Biol. 18, 796-804 (2011)

23. B. Tsang, J. Arsenault, R. M. Vernon, H. Lin, N. Sonenberg, L.-Y. Wang, A. Bah, J. D. Forman-Kay, Phosphoregulated FMRP phase separation models activity-dependent translation through bidirectional control of mRNA granule formation. Proc. Natl. Acad. Sci. U.S.A. 116, 4218-4227 (2019).

24. T. H. Kim, B. Tsang, R. M. Vernon, N. Sonenberg, L. E. Kay, J. D. Forman-Kay, Phosphodependent phase separation of FMRP and CAPRIN1 recapitulates regulation of translation and deadenylation. Science 365, 825-829 (2019).

25. A. M. Krichevsky, K. S. Kosik, Neuronal RNA granules: A link between RNA localization and stimulation-dependent translation. Neuron 32, 683-696 (2001).

26. P. K. Sahoo, S. J. Lee, P. B. Jaiswal, S. Alber, A. N. Kar, S. Miller-Randolph, E. E. Taylor, T. Smith, B. Singh, T. S.-Y. Ho, A. Urisman, S. Chand, E. A. Pena, A. L. Burlingame, C. J. Woolf, M. Fainzilber, A. W. English, J. L. Twiss, Axonal G3BP1 stress granule protein limits axonal mRNA translation and nerve regeneration. Nat. Commun. 9, 3358 (2018).

27. J. López-Erauskin, T. Tadokoro, M. W. Baughn, B. Myers, M. McAlonis-Downes, C. Chillon-Marinas, J. N. Asiaban, J. Artates, A. T. Bui, A. P. Vetto, S. K. Lee, A. V. Le, Y. Sun, M. Jambeau, J. Boubaker, D. Swing, J. Qiu, G. G. Hicks, Z. Ouyang, X.-D. Fu, L. Tessarollo, S.-C. Ling, P. A. Parone, C. E. Shaw, M. Marsala, C. Lagier-Tourenne, D. W. Cleveland, S. Da Cruz, ALS/FTD-linked mutation in FUS suppresses intra-axonal protein synthesis and drives disease without nuclear loss-of-function of FUS. Neuron 100, 816-830.e7 (2018).

28. D. Simsek, G. C. Tiu, R. A. Flynn, G. W. Byeon, K. Leppek, A. F. Xu, H. Y. Chang, M. Barna, The mammalian ribo-interactome reveals ribosome functional diversity and heterogeneity. Cell 169, 1051-1065.e18 (2017).

29. P. Bernabò, T. Tebaldi, E. J. N. Groen, F. M. Lane, E. Perenthaler, F. Mattedi, H. J. Newbery, H. Zhou, P. Zuccotti, V. Potrich, H. K. Shorrock, F. Muntoni, A. Quattrone, T. H. Gillingwater, G. Viero, In vivo translatome profiling in spinal muscular atrophy reveals a role for SMN protein in ribosome biology. Cell Rep. 21, 953-965 (2017).

30. J. C. Darnell, S. J. Van Driesche, C. Zhang, K. Y. S. Hung, A. Mele, C. E. Fraser, E. F. Stone, C. Chen, J. J. Fak, S. W. Chi, D. D. Licatalosi, J. D. Richter, R. B. Darnell, FMRP stalls 
ribosomal translocation on mRNAs linked to synaptic function and autism. Cell 146 247-261 (2011).

31. E. Sanz, L. Yang, T. Su, D. R. Morris, G. S. McKnight, P. S. Amieux, Cell-type-specific isolation of ribosome-associated mRNA from complex tissues. Proc. Natl. Acad. Sci. U.S.A. 106, 13939-13944 (2009).

32. B. Rogelj, L. E. Easton, G. K. Bogu, L. W. Stanton, G. Rot, T. Curk, B. Zupan, Y. Sugimoto, M. Modic, N. Haberman, J. Tollervey, R. Fujii, T. Takumi, C. E. Shaw, J. Ule, Widespread binding of FUS along nascent RNA regulates alternative splicing in the brain. Sci. Rep. 2, 603 (2012).

33. R. Goering, L. I. Hudish, B. B. Guzman, N. Raj, G. J. Bassell, H. A. Russ, D. Dominguez, J. M. Taliaferro, FMRP promotes RNA localization to neuronal projections through interactions between its RGG domain and G-quadruplex RNA sequences. eLife 9, e52621 (2020).

34. S. R. Thomson, S. S. Seo, S. A. Barnes, S. R. Louros, M. Muscas, O. Dando, C. Kirby, D. J. A. Wyllie, G. E. Hardingham, P. C. Kind, E. K. Osterweil, Cell-type-specific translation profiling reveals a novel strategy for treating fragile $X$ syndrome. Neuron $95,550-563 . e 5$ (2017).

35. M. G. Garone, V. Alfano, B. Salvatori, C. Braccia, G. Peruzzi, A. Colantoni, I. Bozzoni, A. Armirotti, A. Rosa, Proteomics analysis of FUS mutant human motoneurons reveals altered regulation of cytoskeleton and other ALS-linked proteins via 3' UTR binding. Sci. Rep. 10, 11827 (2020).

36. E. Ylikallio, R. Pöyhönen, M. Zimon, E. De Vriendt, T. Hilander, A. Paetau, A. Jordanova, T. Lönnqvist, H. Tyynismaa, Deficiency of the E3 ubiquitin ligase TRIM2 in early-onset axonal neuropathy. Hum. Mol. Genet. 22, 2975-2983 (2013).

37. M. Kamelgarn, J. Chen, L. Kuang, H. Jin, E. J. Kasarskis, H. Zhu, ALS mutations of FUS suppress protein translation and disrupt the regulation of nonsense-mediated decay. Proc. Natl. Acad. Sci. U.S.A. 115, E11904-E11913 (2018).

38. M. Sévigny, I. Bourdeau Julien, J. P. Venkatasubramani, J. B. Hui, P. A. Dutchak, C. F. Sephton, FUS contributes to mTOR-dependent inhibition of translation. J. Biol. Chem 295, 18459-18473 (2020).

39. J. C. Mitchell, P. McGoldrick, C. Vance, T. Hortobagyi, J. Sreedharan, B. Rogelj, E. L. Tudor, B. N. Smith, C. Klasen, C. C. J. Miller, J. D. Cooper, L. Greensmith, C. E. Shaw, Overexpression of human wild-type FUS causes progressive motor neuron degeneration in an age- and dose-dependent fashion. Acta Neuropathol. 125, 273-288 (2013).

40. H. Qiu, S. Lee, Y. Shang, W.-Y. Wang, K. F. Au, S. Kamiya, S. J. Barmada, S. Finkbeiner, H. Lui, C. E. Carlton, A. A. Tang, M. C. Oldham, H. Wang, J. Shorter, A. J. Filiano, E. D. Roberson, W. G. Tourtellotte, B. Chen, L.-H. Tsai, E. J. Huang, ALS-associated mutation FUS-R521C causes DNA damage and RNA splicing defects. J. Clin. Invest. 124, 981-999 (2014).

41. C. F. Sephton, A. A. Tang, A. Kulkarni, J. West, M. Brooks, J. J. Stubblefield, Y. Liu, M. Q. Zhang, C. B. Green, K. M. Huber, E. J. Huang, J. Herz, G. Yu, Activity-dependent FUS dysregulation disrupts synaptic homeostasis. Proc. Natl. Acad. Sci. U.S.A. 111, E4769-E4778 (2014).

42. F. Cid-Samper, M. Gelabert-Baldrich, B. Lang, N. Lorenzo-Gotor, R. D. Ponti, L.-A. W. F. M. Severijnen, B. Bolognesi, E. Gelpi, R. K. Hukema, T. Botta-Orfila, G. G. Tartaglia, An integrative study of protein-RNA condensates identifies scaffolding rnas and reveals players in fragile X-associated tremor/ataxia syndrome. Cell Rep. 25, 3422-3434.e7 (2018)

43. J. D. Richter, G. J. Bassell, E. Klann, Dysregulation and restoration of translational homeostasis in fragile X syndrome. Nat. Rev. Neurosci. 16, 595-605 (2015).

44. Y. Tan, C. Sgobio, T. Arzberger, F. Machleid, Q. Tang, E. Findeis, J. Tost, T. Chakroun, P. Gao, M. Höllerhage, K. Bötzel, J. Herms, G. Höglinger, T. Koeglsperger, Loss of fragile X mental retardation protein precedes Lewy pathology in Parkinson's disease. Acta Neuropathol. 139, 319-345 (2020).

45. P. K. Todd, S. Y. Oh, A. Krans, F. He, C. Sellier, M. Frazer, A. J. Renoux, K. Chen, K. M. Scaglione, V. Basrur, K. Elenitoba-Johnson, J. P. Vonsattel, E. D. Louis, M. A. Sutton, J. P. Taylor, R. E. Mills, N. Charlet-Berguerand, H. L. Paulson, CGG repeat-associated translation mediates neurodegeneration in fragile $\mathrm{X}$ tremor ataxia syndrome. Neuron $\mathbf{7 8}$, 440-455 (2013).

46. A. R. Buxbaum, B. Wu, R. H. Singer, Single $\beta$-actin mRNA detection in neurons reveals a mechanism for regulating its translatability. Science 343, 419-422 (2014).

47. H. Y. Park, H. Lim, Y. J. Yoon, A. Follenzi, C. Nwokafor, M. Lopez-Jones, X. Meng, R. H. Singer, Visualization of dynamics of single endogenous mRNA labeled in live mouse Science 343, 422-424 (2014).

48. J. Wang, J.-M. Choi, A. S. Holehouse, H. O. Lee, X. Zhang, M. Jahnel, S. Maharana, R. Lemaitre, A. Pozniakovsky, D. Drechsel, I. Poser, R. V. Pappu, S. Alberti, A. A. Hyman, A molecular grammar governing the driving forces for phase separation of prion-like RNA binding proteins. Cell 174, 688-699.e16 (2018).

49. A. M. Blokhuis, E. J. N. Groen, M. Koppers, L. H. van den Berg, R. J. Pasterkamp, Protein aggregation in amyotrophic lateral sclerosis. Acta Neuropathol. 125, 777-794 (2013).

50. T. Gonatopoulos-Pournatzis, R. Niibori, E. W. Salter, R. J. Weatheritt, B. Tsang, S. Farhangmehr, X. Liang, U. Braunschweig, J. Roth, S. Zhang, T. Henderson, E. Sharma, M. Quesnel-Vallières, J. Permanyer, S. Maier, J. Georgiou, M. Irimia, N. Sonenberg,
J. D. Forman-Kay, A.-C. Gingras, G. L. Collingridge, M. A. Woodin, S. P. Cordes, B. J. Blencowe, Autism-misregulated elF4G microexons control synaptic translation and higher order cognitive functions. Mol. Cell 77, 1176-1192.e16 (2020).

51. J. Humphrey, N. Birsa, C. Milioto, M. McLaughlin, A. M. Ule, D. Robaldo, A. B. Eberle, R. Kräuchi, M. Bentham, A.-L. Brown, S. Jarvis, C. Bodo, M. G. Garone, A. Devoy, G. Soraru, A. Rosa, I. Bozzoni, E. M. C. Fisher, O. Mühlemann, G. Schiavo, M.-D. Ruepp, A. M. Isaacs, V. Plagnol, P. Fratta, FUS ALS-causative mutations impair FUS autoregulation and splicing factor networks through intron retention. Nucleic Acids Res. 48, 6889-6905 (2020).

52. M. G. Garone, N. Birsa, M. Rosito, F. Salaris, M. Mochi, V. de Turris, R. R. Nair, T. J. Cunningham, E. M. C. Fisher, P. Fratta, A. Rosa, RNA-binding protein network alteration causes aberrant axon branching and growth phenotypes in FUS ALS mutant motoneurons. bioRxiv, 2020.08.26.268631 , (2020).

53. G. E. Tyzack, R. Luisier, D. M. Taha, J. Neeves, M. Modic, J. S. Mitchell, I. Meyer, L. Greensmith, J. Newcombe, J. Ule, N. M. Luscombe, R. Patani, Widespread FUS mislocalization is a molecular hallmark of amyotrophic lateral sclerosis. Brain 142. 2572-2580 (2019).

54. T. Lashley, J. D. Rohrer, R. Bandopadhyay, C. Fry, Z. Ahmed, A. M. Isaacs, J. H. Brelstaff, B. Borroni, J. D. Warren, C. Troakes, A. King, S. Al-Saraj, J. Newcombe, N. Quinn, K. Ostergaard, H. D. Schrøder, M. Bojsen-Møller, H. Braendgaard, N. C. Fox, M. N. Rossor, A. J. Lees, J. L. Holton, T. Revesz, A comparative clinical, pathological, biochemical and genetic study of fused in sarcoma proteinopathies. Brain 134, 2548-2564 (2011).

55. M. Neumann, R. Rademakers, S. Roeber, M. Baker, H. A. Kretzschmar, I. R. A. Mackenzie, A new subtype of frontotemporal lobar degeneration with FUS pathology. Brain 132, 2922-2931 (2009).

56. Y. Zhou, S. Liu, G. Liu, A. Öztürk, G. G. Hicks, ALS-associated FUS mutations result in compromised FUS alternative splicing and autoregulation. PLOS Genet. 9, e1003895 (2013).

57. C. Fallini, G. J. Bassell, W. Rossoll, High-efficiency transfection of cultured primary motor neurons to study protein localization, trafficking, and function. Mol. Neurodegener. 5, 17 (2010).

58. J. Lenzi, R. De Santis, V. de Turris, M. Morlando, P. Laneve, A. Calvo, V. Caliendo, A. Chiò, A. Rosa, I. Bozzoni, ALS mutant FUS proteins are recruited into stress granules in induced pluripotent stem cell-derived motoneurons. Dis. Model. Mech. 8, 755-766 (2015).

59. R. De Santis, V. Alfano, V. de Turris, A. Colantoni, L. Santini, M. G. Garone, G. Antonacci, G. Peruzzi, E. Sudria-Lopez, E. Wyler, J. J. Anink, E. Aronica, M. Landthaler, R. J. Pasterkamp, I. Bozzoni, A. Rosa, Mutant FUS and ELAVL4 (HuD) aberrant crosstalk in amyotrophic lateral sclerosis. Cell Rep. 27, 3818-3831.e5 (2019).

60. L. Restani, F. Giribaldi, M. Manich, K. Bercsenyi, G. Menendez, O. Rossetto, M. Caleo, G. Schiavo, Botulinum neurotoxins $A$ and $E$ undergo retrograde axonal transport in primary motor neurons. PLOS Pathog. 8, e1003087 (2012).

61. T. G. Moens, T. Niccoli, K. M. Wilson, M. L. Atilano, N. Birsa, L. M. Gittings, B. V. Holbling, M. C. Dyson, A. Thoeng, J. Neeves, I. Glaria, L. Yu, J. Bussmann, E. Storkebaum, M. Pardo, J. S. Choudhary, P. Fratta, L. Partridge, A. M. Isaacs, C9orf72 arginine-rich dipeptide proteins interact with ribosomal proteins in vivo to induce a toxic translational arrest that is rescued by elF1 A. Acta Neuropathol. 137, 487-500 (2019).

62. E. Danielson, S. H. Lee, SynPAnal: Software for rapid quantification of the density and intensity of protein puncta from fluorescence microscopy images of neurons. PLOS ONE 9, e115298 (2014)

63. A. D. Fellows, E. R. Rhymes, K. L. Gibbs, L. Greensmith, G. Schiavo, IGF1R regulates retrograde axonal transport of signalling endosomes in motor neurons. EMBO Rep. 21, e49129 (2020).

64. J.-Y. Tinevez, N. Perry, J. Schindelin, G. M. Hoopes, G. D. Reynolds, E. Laplantine, S. Y. Bednarek, S. L. Shorte, K. W. Eliceiri, TrackMate: An open and extensible platform for single-particle tracking. Methods 115, 80-90 (2017).

65. T. Shigeoka, H. Jung, J. Jung, B. Turner-Bridger, J. Ohk, J. Q. Lin, P. S. Amieux, C. E. Holt, Dynamic axonal translation in developing and mature visual circuits. Cell 166, 181-192 (2016).

66. A. Dobin, C. A. Davis, F. Schlesinger, J. Drenkow, C. Zaleski, S. Jha, P. Batut, M. Chaisson, T. R. Gingeras, STAR: Ultrafast universal RNA-seq aligner. Bioinformatics 29, 15-21 (2013).

67. J. M. Mudge, J. Harrow, Creating reference gene annotation for the mouse C57BL6/J genome assembly. Mamm. Genome 26, 366-378 (2015)

68. M. I. Love, W. Huber, S. Anders, Moderated estimation of fold change and dispersion for RNA-seq data with DESeq2. Genome Biol. 15, 550 (2014).

69. J. Reimand, M. Kull, H. Peterson, J. Hansen, J. Vilo, g:Profiler-A web-based toolset for functional profiling of gene lists from large-scale experiments. Nucleic Acids Res. 35, W193-W200 (2007).

70. R. G. Cavalcante, M. A. Sartor, annotatr: Genomic regions in context. Bioinformatics 33, 2381-2383 (2017).

71. Carlson, org.Mm.eg.db: Genome wide annotation for Mouse. R package version 3.8.2. (2019). 
72. Carlson, Maintainer, TxDb.Mmusculus.UCSC.mm9.knownGene: Annotation package for TxDb object(s). R package version 3.2.2. (2015), doi:10.18129/B9.bioc.TxDb.Mmusculus. UCSC.mm9.knownGene.

73. G. Yu, L.-G. Wang, Y. Han, Q.-Y. He, clusterProfiler: An R package for comparing biological themes among gene clusters. OMICS 16, 284-287 (2012).

Acknowledgments: We thank D. Dormann and M.-D. Ruepp for discussion, E. Spaulding and R. Burgess for discussion and technical advice on the RiboTag method, J. Sleigh for critical reading of this manuscript, and all members of the Fratta and Schiavo laboratories for discussion. Funding: This study was supported by the following: MRC/MNDA Lady Edith Wolfson Fellowship (to P.F); NIHR-UCLH Biomedical Research Centre (to P.F.); U.K. Medical Research Council (MR/M008606/1 and MR/S006508/1 to P.F. and MR/S022708/1 to E.M.C.F. and P.F.); Motor Neurone Disease Association (885-792 to P.F.); Rosetrees Trust (to P.F. and E.M.C.F.); Wellcome Trust Senior Investigator Award (107116/Z/15/Z to G.S.); U.K. Dementia Research Institute Foundation award (UKDRI-1005 to G.S.); Canadian Institutes of Health Research Foundation (grant no.148375 to J.D.F.-K.); and AriSLA Foundation (AxRibALS to G.V.). Author contributions: Conceptualization, P.F. Investigation, N.B., A.M.U., M.G.G., B.T., F.M., M.P. O.G.W., and G.V. Data curation, J.H. and S.J. Formal analysis, A.M.U. and N.B. Writing-origina draft, N.B., P.F., and A.M.U. Writing-review and editing, N.B., A.M.U., M.G.G., B.T., F.M., P.A.C., J.H., E.M.C.F., A.R., G.V., J.D.F.-K., G.S., and P.F. Resources, C.B., R.F.d.I.F., B.T., P.A.C., M.L.N., and A.D. Funding acquisition, P.F., J.D.F.-K., and G.V. Supervision, P.F., J.D.F.-K., and G.S. Competing interests: The authors declare that they have no competing interests. Data and materials availability: All data needed to evaluate the conclusions in the paper are present in the paper and/or in the Supplementary Materials. Raw RNA-sequencing data from the Ribotag datasets (input and Ribo IP) were deposited as the NCBI BioProject with accession code: PRJNA694181 and ID: 694181

Submitted 24 November 2020

Accepted 3 June 2021

Published 21 July 2021

$10.1126 /$ sciadv.abf 8660

Citation: N. Birsa, A. M. Ule, M. G. Garone, B. Tsang, F. Mattedi, P. A. Chong, J. Humphrey, S. Jarvis, M. Pisiren, O. G. Wilkins, M. L. Nosella, A. Devoy, C. Bodo, R. F. de la Fuente, E. M. C. Fisher, A. Rosa, G. Viero, J. D. Forman-Kay, G. Schiavo, P. Fratta, FUS-ALS mutants alter FMRP phase separation equilibrium and impair protein translation. Sci. Adv. 7, eabf8660 (2021). 


\section{ScienceAdvances}

\section{FUS-ALS mutants alter FMRP phase separation equilibrium and impair protein translation}

Nicol Birsa, Agnieszka M. Ule, Maria Giovanna Garone, Brian Tsang, Francesca Mattedi, P. Andrew Chong, Jack Humphrey, Seth Jarvis, Melis Pisiren, Oscar G. Wilkins, Micheal L. Nosella, Anny Devoy, Cristian Bodo, Rafaela Fernandez de la Fuente, Elizabeth M. C. Fisher, Alessandro Rosa, Gabriella Viero, Julie D. Forman-Kay, Giampietro Schiavo and Pietro Fratta

Sci Adv 7 (30), eabf8660.

DOI: $10.1126 /$ sciadv.abf8660

ARTICLE TOOLS

SUPPLEMENTARY

MATERIALS

REFERENCES

PERMISSIONS http://advances.sciencemag.org/content/7/30/eabf8660

http://advances.sciencemag.org/content/suppl/2021/07/19/7.30.eabf8660.DC1

This article cites 70 articles, 13 of which you can access for free http://advances.sciencemag.org/content/7/30/eabf8660\#BIBL

http://www.sciencemag.org/help/reprints-and-permissions

Science Advances (ISSN 2375-2548) is published by the American Association for the Advancement of Science, 1200 New York Avenue NW, Washington, DC 20005. The title Science Advances is a registered trademark of AAAS.

Copyright @ 2021 The Authors, some rights reserved; exclusive licensee American Association for the Advancement of Science. No claim to original U.S. Government Works. Distributed under a Creative Commons Attribution License 4.0 (CC $\mathrm{BY})$. 